\title{
WAVEFORM DESIGN WITH TIME AND FREQUENCY CONSTRAINTS FOR OPTIMAL DETECTION OF ELASTIC OBJECTS
}

by

\section{Brandon M. Hamschin}

B.S. in Electrical Engineering, Pennsylvania State University, 2005 B.S. in Mathematics, Pennsylvania State University, 2005

Submitted to the Graduate Faculty of the Swanson School of Engineering in partial fulfillment of the requirements for the degree of Master of Science

University of Pittsburgh 2011 


\section{UNIVERSITY OF PITTSBURGH \\ SWANSON SCHOOL OF ENGINEERING}

This thesis was presented

by

Brandon M. Hamschin

It was defended on

March 28, 2010

and approved by

Patrick J. Loughlin, PhD, Professor, Depts. of Bioengineering and Electrical and Computer Engineering

Amro El-Jaroudi, PhD, Associate Professor, Dept. of Electrical and Computer Engineering Steven Jacobs, PhD, Assistant Professor, Dept. of Electrical and Computer Engineering Thesis Advisor: Patrick J. Loughlin, PhD, Professor, Depts. of Bioengineering and Electrical and Computer Engineering 
Copyright (c) by Brandon M. Hamschin

2011 


\title{
WAVEFORM DESIGN WITH TIME AND FREQUENCY CONSTRAINTS FOR OPTIMAL DETECTION OF ELASTIC OBJECTS
}

\author{
Brandon M. Hamschin, M.S. \\ University of Pittsburgh, 2011
}

In active sonar, the goal is to learn about an object or environment by transmitting a sound and processing the echo. The sound we choose to transmit will determine what we learn about the object, much like the choice of question we ask a person will determine what we learn from them. Thus, designing the best (i.e. optimal) transmit waveform is a longstanding area of research that remains active since different environments and ever evolving operational objectives weigh heavily on how we define optimality.

In this work we extend a recent result by Kay that gives the optimal transmit signal that maximizes the probability of detecting an elastic object in the presence of Gaussian reverberation and additive Gaussian interference. Kay's solution specifies the spectral magnitude for the optimal transmit waveform, and hence there is an unlimited number of "optimal" waveforms that can be transmitted, all with the same spectral magnitude but differing in terms of time domain characteristics such as duration and peak power. We extend Kay's approach in order to obtain a unique optimal waveform by incorporating time-domain constraints into two optimization-based problem formulations. These two problem formulations lead to new and complementary signal design approaches that impose temporal duration constraints while preserving, to varying degrees, the optimality inherent in the spectral magnitude. 


\section{TABLE OF CONTENTS}

1.0 INTRODUCTION . . . . . . . . . . . . . . . . . . . . . . . . . . 1

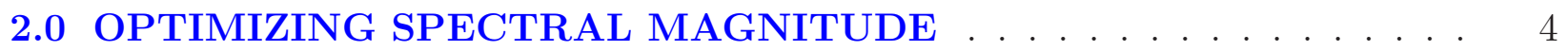

2.1 Introduction . . . . . . . . . . . . . . . . . . . . . 4

2.2 The Optimal Spectral Density . . . . . . . . . . . . . . . 5

2.3 Examples . . . . . . . . . . . . . . . . . . . 9

2.3.1 Ex 1: Point Target in White Noise and White Reverberation . . . 10

2.3.2 Ex 2: Point Target in Colored Noise and White Reverberation . . . 12

2.3.3 Ex 3: Elastic Target in White Noise and White Reverberation ... 14

2.3.4 Ex 4: Elastic Target in Colored Noise and White Reverberation . . 16

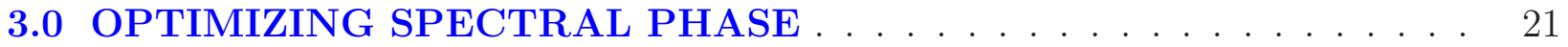

3.1 Introduction . . . . . . . . . . . . . . . . . . . 21

3.2 The Optimal Spectral Phase . . . . . . . . . . . . . . . . . . . . . . . 22

3.2.1 The Minimum Duration Signal . . . . . . . . . . . . . . 23

3.2 .2 The Maximum Duration Signal . . . . . . . . . . . . . . . 24

3.2.3 Blending the Maximum and Minimum Duration Solutions . . . . 25

3.3 Examples . . . . . . . . . . . . . . . . . . . . . . 26

4.0 OPTIMIZING TEMPORAL CONCENTRATION . . . . . . . . . . . 29

4.1 Introduction . . . . . . . . . . . . . . . . . . . 29

4.2 The Classical Slepian Problem . . . . . . . . . . . . . . . . . . . . . 31

4.3 The Modified Slepian Concentration Problem _ . . . . . . . . . . . . 33

4.3.1 Mathematical Formulation: Discrete Time/Continuous Frequency . 33

4.3.2 Mathematical Formulation: Discrete Time/Discrete Frequency . . . 34 
4.3.3 Mathematical Formulation: Nonlinear Program . . . . . . . . . . 34

4.3.4 Connection to the Minimum Duration Solution . . . . . . . . 36

4.4 Examples . . . . . . . . . . . . . . . . . . . . . . 37

4.4.1 Ex 1: Equivalence of Classical and Modified Formulations as $\epsilon \rightarrow \infty \quad 37$

4.4.2 Ex 2: Solution to Modified Formulation for $\epsilon=0.01 \ldots \ldots$. . . . 38

4.4.3 Ex 3: Solution to Modified Formulation for $\epsilon=0.3 \ldots \ldots$

4.4.4 Ex 4: Solution to Modified Formulation for $\epsilon=3.0 \quad \ldots \ldots$. . . 40

5.0 CONCLUSIONS AND FUTURE DIRECTIONS . . . . . . . . . . . 43

APPENDIX A. LINEAR/MINIMUM PHASE SIGNAL PROPERTIES . . 45

APPENDIX B. MULTITARGET WAVEFORM DESIGN . . . . . . . . . . . 48

B.1 Multitarget Waveform Design for Optimal Detection . . . . . . . . . 49

B.1.1 Initialization . . . . . . . . . . . . . . . . . . . 50

B.1.2 Simulation Results . . . . . . . . . . . . . . . . 51

B.2 Multitarget Waveform Design for Optimal Classification . . . . . . . . 54

APPENDIX C. EXTENSIONS TO NONSTATIONARY ENVIRONMENTS 56

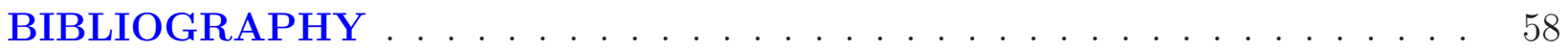




\section{LIST OF TABLES}

1 Material properties of the shell and surrounding environment. . . . . . . . 15

2 Duration and Peak-to-Average Energy Results . . . . . . . . . . . . 26 


\section{LIST OF FIGURES}

1 Model of received signal $x(t)$, and associated detector $D(x) \ldots \ldots$

2 Example 1 - Point target in white noise and white reverberation . . . . . . 11

3 Example 2 - Point target in colored noise and white reverberation . . . . . 13

4 Diagram of Spherical Target used in Examples 3 and 4 . . . . . . . . . . 15

5 Example 3 - Elastic target in white noise and white reverberation . . . . . 17

6 Example 3 - Relationship between noise, target, and optimal ESD . . . . . 18

7 Example 4 - Elastic target in colored noise and white reverberation . . . . . . 19

8 Example 4 - Relationship between noise, target, and optimal ESD . . . . . 20

9 Examples - Spectrogram of each spectral phase based signal design . . . . . . 28

10 Example 1 - Equivalence of Classical and Modified Methods for $\epsilon \rightarrow \infty$. . 37

11 Example 2 - Signal Design Results for $\epsilon=0.01$. . . . . . . . . . . . 39

12 Example 3 - Signal Design Results for $\epsilon=0.3$. . . . . . . . . . . . . . . . . 41

13 Example 4 - Signal Design Results for $\epsilon=3.0$. . . . . . . . . . . . . . . 42

14 Minimum Phase signal design . . . . . . . . . . . . . . . . . . . . . . . 47

15 Multitarget Waveform Design-Spectra . . . . . . . . . . . . . . 52

16 Multitarget Waveform Design-Performance . . . . . . . . . . . . 53 


\subsection{INTRODUCTION}

Transmit waveform design for RADAR and SONAR has a long history and is an area that continues to receive active interest, including biomimetic and optimization-based approaches (e.g., $[1,2,3,4,5,6,7,8,9]$ ). In this thesis, we consider the design of transmit waveforms for optimizing the detection of underwater objects, particularly elastic objects ${ }^{1}$, for which the assumption of a point target response is not accurate, in that the received waveform is not simply a time-delayed and attenuated replica of the transmit waveform. We consider a further level of complexity by assuming that the receiver must contend with both additive noise that is independent of the transmit signal, as well as signal-dependent noise, such as occurs with reverberation and clutter. Accordingly, as we will see, the optimal detector is not simply a classical matched filter. Rather, the optimal detector and the waveform that maximizes the probability of detection are coupled, both of which are dependent on the target response and the statistical properties of the environment.

To incorporate these more complex assumptions into new waveform design approaches, we utilize and build on some recent developments in the field, particularly those of Kay $[4,5]$. Although these and other approaches [2] consider different criteria for optimizing detection, a commonality in the solutions is that each specifies only the magnitude spectrum (the power spectrum or energy density spectrum) of the transmit waveform. The basic result is to design the magnitude spectrum so that there is energy in frequency bands where the target response is large relative to the effects of all sources of interference. Accordingly, because the magnitude spectrum does not uniquely specify the time domain signal, there is an unlimited number of optimal transmit waveforms with the same magnitude spectrum.

\footnotetext{
${ }^{1}$ In contrast to a point refelctor, an elastic object generally has a complicated frequency response whereby different excitation frequencies result in varying levels of reflected acoustic energy.
} 
So, in order to uniquely specify an optimal time-domain transmit waveform one must not only specify the spectral magnitude but also the spectral phase for which, depending on the application, a number of approaches can be taken. Specifically, in radar applications one is usually forced, due to hardware constraints, to design signals with constant modulus (i.e. signals free of amplitude modulation). Consequently, such designs must encode all spectral magnitude requirements into the temporal phase of the transmit signal $[10,11,12]$.

In this work we design signals that possess optimal temporal and spectral properties but are free of the constant modulus requirement. In particular, we consider transmit waveform designs based on two problem formulations. In the first formulation [13], we directly design spectral phase functions based on the desire to minimize or maximize the duration of the waveform, subject to the optimal spectral magnitude criterion developed by Kay in [4] for point targets and extended to elastic targets in $[5,13]$. The resultant waveforms give the designer the freedom to choose signals with short duration but also high peak energy or signals with lower peak energy and longer duration, while maintaining optimal detection performance. A solution that blends these two extremes is also given.

In the second formulation the goal is to design time domain signals that are maximally concentrated in a given time interval at the cost of suboptimal detection performance. The mathematical formulation of the problem is motivated by the work of Slepian, Pollak, and Landau $[14,15,16,17]$, who in a series of now classic papers first formulated and solved the so-called concentration problem. In our work we formulate the classical concentration problem as a constrained nonlinear optimization problem, to which we add constraints that incorporate the optimal spectral magnitude from $[4,5,13]$. Solving this modified optimization problem produces a time-domain signal that is real, maximally concentrated in the discrete-time interval $(0, N-1)$, and has a magnitude spectrum that is arbitrarily close, in the least squares sense, to that which maximizes detection performance. The trade-off is, for a fixed $N$ that is sufficiently small, the closer we force the spectrum of the designed signal to that of the optimal spectrum the more energy leakage we find in the samples outside the $(0, N-1)$ interval. 
To develop our central ideas, the remainder of this thesis is organized as follows. In Chapter 2 we overview the main results from [4] and summarize their extension to the elastic target case. Next, in Chapter 3, we design time domain signals having not only the optimal magnitude spectrum from Chapter 2 but also optimal duration properties, by designing the spectral phase. In Chapter 4 we relax, to varying degrees, the spectral magnitude requirement from Chapter 2 in order to design signals that are maximally concentrated in a specified time interval. In each of these chapters we motivate the problem formulation intuitively, derive the optimal waveform mathematically, and analyze system performance analytically or via simulation. Finally, we conclude with a summary of the main results and suggest future research directions. 


\subsection{OPTIMIZING SPECTRAL MAGNITUDE}

\subsection{INTRODUCTION}

People are uniquely equipped to inquire and process responses, and do so with remarkable skill. Yet, the real world often poses challenges that confound our efforts to make proper sense of what we perceive. For example, consider a conversation with a friend near a busy street. The communication is likely to be subject to vehicular noise or the extraneous conversations of bystanders, the character of which is independent of the conversation. Furthermore, suppose the conversation is in a location where nearby buildings produce echos of the conversation. Unlike the vehicular noise, this form of interference is dependent on the speakers and the characteristics of the surroundings. Each of these interference sources are likely to degrade the interchange of information between speakers and, consequently, make it difficult to understand the message. Remarkably, the human brain has an extraordinary ability to filter out extraneous interference and focus on the desired source.

Interference sources of this nature are not only present in our everyday lives but are also prevalent in underwater acoustics. In particular, sonar systems are subject to ambient ocean noise from biologic sources (e.g. whale calls or snapping shrimp), manmade sources (e.g. merchant ships), and self noise generated by sonar system hardware[18]. These interference sources, though troublesome, are not usually dependent on the sound pulse transmitted by the sonar system, similar in kind to street noise from our conversation analogy. Additionally, sonar receivers must contend with interference that is a result of the transmitted waveform. Known as reverberation, such interference is analogous to the building echos in the conversation analogy. More specifically, reverberation is energy present at the receiver that is due to reflections of the transmitted signal from bodies that are not of interest to the sonar task. 
In this chapter we present a signal model that not only accounts for these interference sources but also incorporates a model of the target we wish to detect. The objective is then to develop an optimal method for detecting the target of interest and then to derive a waveform that supports this task in an optimal way.

\subsection{THE OPTIMAL SPECTRAL DENSITY}

We denote the transmit waveform by $s(t)$, and its Fourier spectrum as $S(f)$ by ${ }^{1}$

$$
\begin{aligned}
s(t) & =\sqrt{T} \int_{-W / 2}^{W / 2} S(f) e^{j 2 \pi t} d f \\
S(f) & =\frac{1}{\sqrt{T}} \int_{-T / 2}^{T / 2} s(t) e^{-j 2 \pi f t} d t
\end{aligned}
$$

where $T$ is the observation interval of the received signal and $-W / 2 \leq f \leq W / 2$ is the effective bandwidth. It is also convenient to express the spectrum in terms of its amplitude $B(f)$ and phase $\psi(f)$,

$$
S(f)=B(f) e^{j \psi(f)}
$$

In the remainder of this section we summarize the main results from Kay $[4,5]$ that are central to the considerations of this chapter as well as those that follow. In [4], Kay treats the problem of designing a signal that is optimal for detecting a point target in reverberation. This development assumes a single transmitter and a single receiver.

In [5], Kay extends his results to the single transmitter and multiple receiver case. In addition to extending the results from [4] to account for multiple receivers, this treatment considers a model of the target that is more general, which in the context of SONAR would include an elastic target. The optimal detector is derived under these more general assumptions, in the context of the Neyman-Pearson criteria for optimality. Subsequently the performance of the detector is derived. Unlike the single receiver case, the expression that is

\footnotetext{
${ }^{1}$ This definition of the Fourier Transform is chosen for consistency with [4]
} 


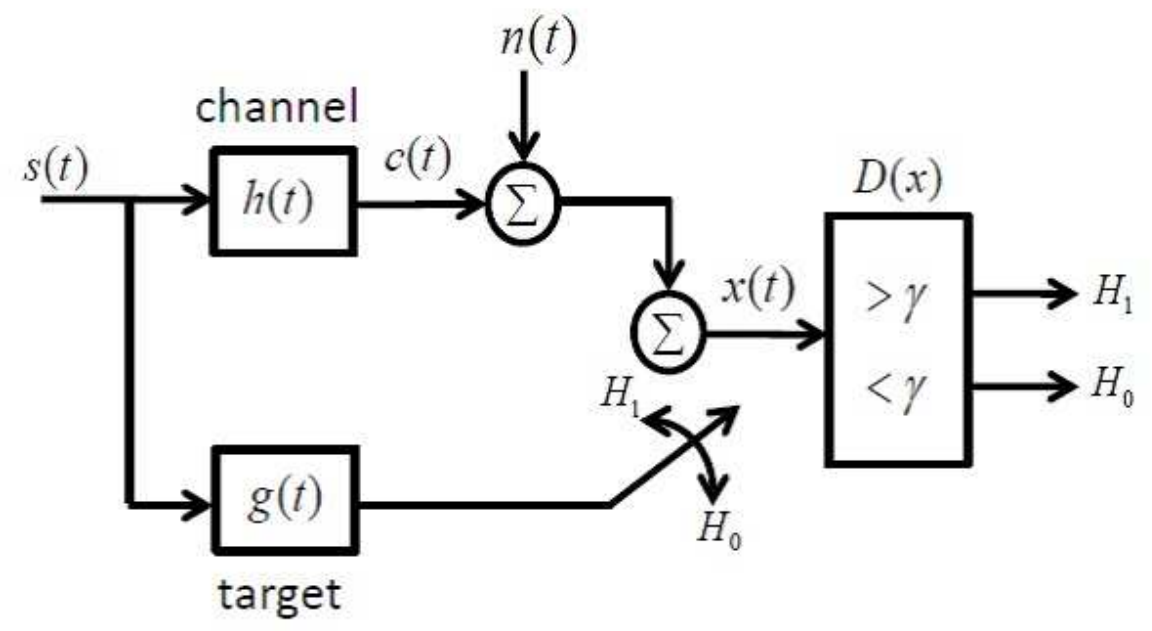

Figure 1: Model of received signal $x(t)$, and associated detector $D(x) . s(t)$ is the transmitted signal; $h(t)$ is the impulse response of a random LTI filter that models channel interference induced by the transmit signal; $g(t)$ is the deterministic impulse response of the object to be detected; and $n(t)$ represents ambient noise. 
obtained for the probability of detection, $P_{D}$, as a function of probability of false alarm, $P_{F A}$, does not readily admit a technique for its maximization over all transmit signals. To circumvent this difficulty, Kay considers an alternative design criterion, divergence, that leads to fruitful results for this more general case.

For the purposes of this chapter we are interested in the results for the case of a single transmitter, single receiver, and a single deterministic elastic target with our aim being obtaining a closed form expression for the optimal magnitude spectrum, $B_{\text {opt }}(\omega)$. With reference to Figure 1, let $g(t)$ be a deterministic LTI model of the elastic target (i.e. the impulse response), $h(t)$ be a Gaussian random process with power spectral density (PSD) $P_{h}(f)$ modeling the reverberation (i.e. signal induced noise), and $n(t)$ be a Gaussian random process with $\operatorname{PSD} P_{n}(f)$ modeling additive system noise and environmental interference. The detector that maximizes the probability of detecting $g(t)$ for a fixed false alarm rate $P_{F A}$ is given by [5]

$$
D(X)=\left|T \int_{-W / 2}^{W / 2} \frac{X(f) S^{*}(f) G^{*}(f)}{P_{h}(f) T|S(f)|^{2}+P_{n}(f)} d f\right|^{2}
$$

where the received signal is $x(t)$ which has Fourier transform $X(\omega), s(t)$ is the known transmit signal with Fourier transform $S(f)$, and $G(f)$ (i.e., the frequency response of the object to be detected) is the Fourier transform of $g(t)$. The null hypothesis $H_{0}$ is rejected - i.e., a decision that the object is present is made - when $D(X)$ exceeds the threshold

$$
\gamma=-\sigma_{0}^{2} \log \left(P_{F A}\right)
$$

where

$$
\sigma_{0}^{2}=\int_{-W / 2}^{W / 2} \frac{T B^{2}(f)|G(f)|^{2}}{P_{h}(f) T B^{2}(f)+P_{n}(f)} d f
$$

Also, it can be shown that the performance of the detector in (2.4) is given by [4]

$$
P_{D}=P_{F A}^{\frac{1}{1+\sigma_{0}^{2}}}
$$

where $P_{D}$ denotes the probability of detection (i.e. deciding the target is present when it actually is). This simple but important equation shows that for a fixed $P_{F A}$ one can increase $P_{D}$ by making $\sigma_{0}^{2}$ large. Finally, it is this observation and by following the point target 
derivation in [4] that we arrive at the magnitude squared spectrum of $s(t)$ that maximizes (2.7) for a fixed $P_{F A}$

$$
T B_{\text {opt }}^{2}(f)=\max \left(\frac{\lambda^{-1 / 2} \sqrt{P_{n}(f)|G(f)|^{2}}-P_{n}(f)}{P_{h}(f)}, 0\right)
$$

where $\lambda$ is a constant, typically obtained numerically, that constrains the total energy of the transmit signal to some specified level, $\int_{-W / 2}^{W / 2} T B_{o p t}^{2}(f) d f=E$. As we will see later, the value of $\lambda$ directly relates to the spectral regions of the optimal waveform that have nonzero energy.

The solution given in (2.8) is obtained by noting that, for a fixed $P_{F A},(2.7)$ is a monotonically increasing function of $\sigma_{0}^{2}$. Since $\sigma_{0}^{2}$ is a function of $B^{2}(f)$ it becomes clear that one should choose $B^{2}(\omega)$ that maximizes (2.6). Fortunately, since the integrand of (2.6) can be shown to be a concave functional of $B^{2}(f)$, a unique $B^{2}(f)$ can be found by maximizing $\sigma_{0}^{2}$. $B_{\text {opt }}^{2}(f)$ is then obtained by treating the integrand as a scalar function of $T B^{2}$ and maximizing it (the integrand) by standard Lagrange multiplier techniques. Though the concavity of the integrand of (2.6) simplifies the theory necessary to find a solution and ensures its uniqueness, the analysis to obtain the solution, while imposing the non-negativity and energy constraints, is not trivial.

Before summarizing what we learned in this chapter, an important trait of the procedure by which the optimal signal was derived should be highlighted. Specifically, the approach was to determine how performance (2.7) of the optimal processing structure (2.4) related to the probing signal. Once this mathematical link was formed (i.e. (2.6) and (2.7)), measures were taken to determine the signal that maximizes the performance of the already optimal processing structure. In other words, with or without signal design the detector in $D(X)$ and its associated threshold $\gamma$ are optimal in the Neyman-Pearson sense. But using (2.8) as the spectrum of the transmitted signal allows one to essentially further optimize optimal performance. This trait is encountered repeatedly in approaches to designing optimal signals for detection [19, pp. 103, 108-112], [20, 9] or classification [21]. 
In summary, if we are able to characterize the spectral properties of the target and the environment, we maximize the probability of detection by transmitting a signal whose magnitude spectrum is given by $B_{\text {opt }}(f)$. It is clear, then, that under the above modeling assumptions, optimal detection performance is independent of the spectral phase of the transmit waveform, and hence there is an unlimited number of possible time domain waveforms that are "optimal" in this regard - an observation that motivates the content of Chapter 3.

\subsection{EXAMPLES}

In this section we cover four examples that highlight some interesting characteristics of the optimal transmit signal spectrum. The examples progress from simplest to most complex.

Before delving into the examples, we derive an interesting result from (2.8) by asking the simple question: What regions in the optimal spectrum have zero energy? Because of the

max operation in (2.8) the answer is when $\lambda^{-1 / 2} \sqrt{P_{n}(f)|G(f)|^{2}}-P_{n}(f)<0$. This condition corresponds to the following relationship between $\lambda$ and the ratio of the noise spectrum to the target spectrum

$$
\frac{P_{n}(f)}{|G(f)|^{2}}>\frac{1}{\lambda}
$$

This inequality implies that in regions of the frequency spectrum where the ratio of the noise power to the target energy exceeds a threshold, the optimal transmit waveform has zero energy. This result is even more interesting since, as stated in the paragraph following (2.8), $\lambda$ is a parameter that is dictated by the total energy constraint on $s(t)$. So, loosely speaking, regions where the noise spectrum is large relative to the responsiveness of the target are avoided by the optimal design; an intuitively pleasing result. One may then ask what effect does the reverberation spectrum have on the signal, given that it does not show up in (2.9)? In contrast to the noise spectrum, $P_{h}(f)$ contributes only to the strength of the optimal transmit spectrum in regions where $P_{n}(f) /|G(f)|^{2}$ is small. Specifically, for the regions where $P_{n}(f) /|G(f)|^{2}<1 / \lambda$, the energy in the transmit signal at those frequencies is scaled by $1 / P_{h}(f)$. Thus, more energy is placed where channel energy is low. 


\subsubsection{Ex 1: Point Target in White Noise and White Reverberation}

This example illustrates how energy is allocated in frequency for the optimal transmit waveform when we assume the object to be detected acts as a point reflector (i.e. its impulse response is a single delta function and therefore $G(f)=1$ ). We also assume that the additive noise process is white with $P_{n}(f)=1$ and the reverberation process is white with $P_{h}(f)=1$ over $-W / 2 \leq f \leq W / 2$ with $W=2, T=1$, and $E=1 / 8$. A white noise process is something familiar to us but perhaps the idea of a white reverberation process is somewhat less common. A reverberation process that is white corresponds to an environment where, if there were no target, on average the transmitted waveform would be reflected back to the receiver with equal attenuation at all frequencies. With respect to reverberation effects, a white reverberation process represents the worst case scenario since even though there is no target present to reflect the transmitted signal (under the null hypothesis $H_{0}$ ) the received signal is the transmitted signal plus noise, which is a disastrous situation if a simple matched filter is employed.

Given the ubiquity of the Linear Frequency Modulated (LFM) signal in sonar applications, Figure 2 (Top) shows a very interesting result. Specifically, we see that when the noise $P_{n}(f)$, reverberation $P_{h}(f)$, and target $|G(f)|$, respond uniformly over frequency (i.e. have flat spectra) the spectrum of the optimal transmit waveform is identical to the spectrum of a LFM waveform, given by

$$
T\left|S_{L F M}(f)\right|^{2}=\frac{E}{W}
$$

So, the widespread popularity of the LFM signal is justified within the modeling assumptions represented by Figure 1 in that if nothing is known about the target or the interference environment, modeling their effects with a white processes is reasonable and results in the LFM signal being optimal. Fig. 2 (Bottom) compares the ROC curves (on a semi-log scale) of the optimal signal and an LFM signal. Given that the optimal magnitude spectrum has an identical spectrum to that of an LFM signal in this case their ROC curves are identical. 

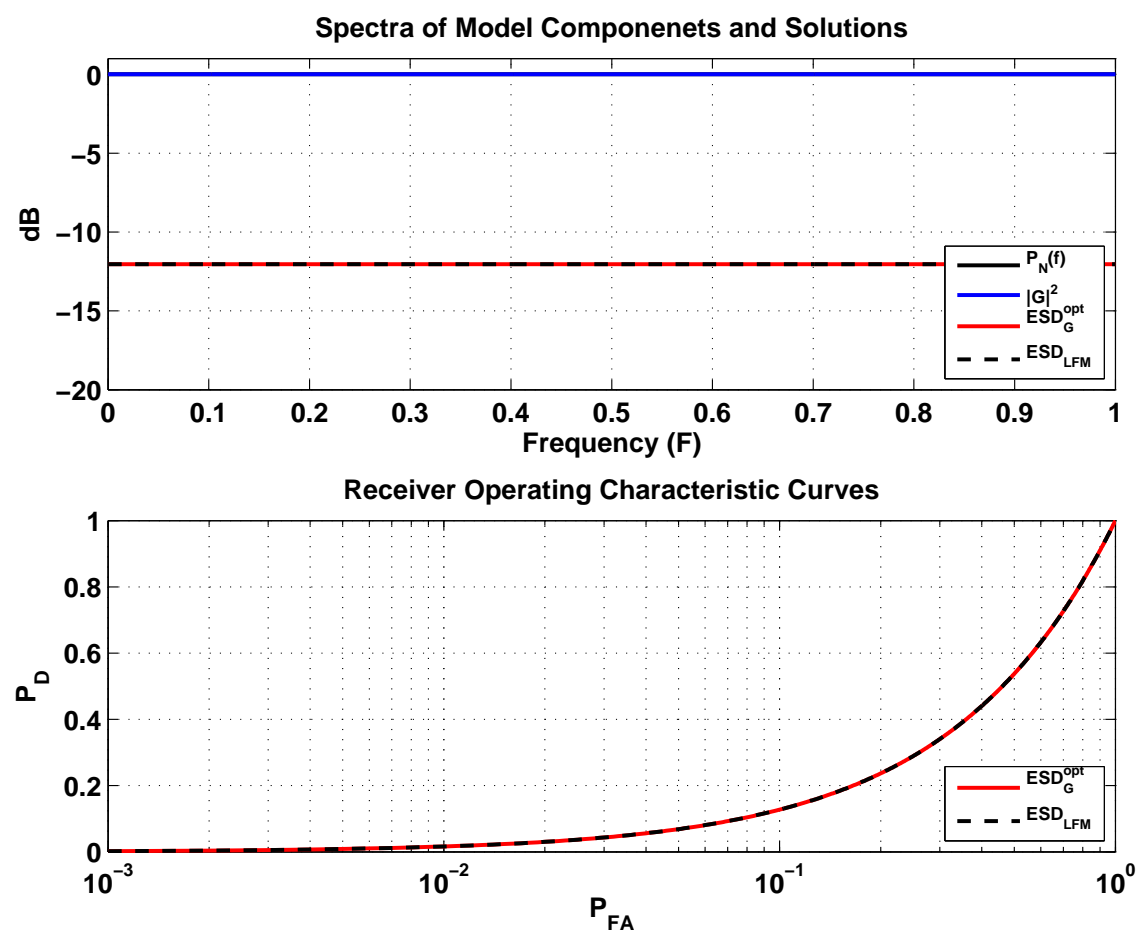

Figure 2: Example 1 - Point target in white noise and white reverberation. (TOP) Spectrum of optimal signal (red), LFM (dashed black), Target response(solid blue), and Noise spectrum (solid black), (BOTTOM) ROC curves for optimal design (red) and LFM (black dashed) 


\subsubsection{Ex 2: Point Target in Colored Noise and White Reverberation}

In this example, which is taken from [4], we again consider a scenario where the target is a point target and the reverberation is white, but now the interference noise is colored. In particular, we let $P_{N}(f)=P_{I}(f)+N_{0}$ where $N_{0}=1$ and

$$
P_{I}(f)=\sum_{i=0}^{3} P_{i} e^{\frac{-\left(f-f_{i}\right)^{2}}{2 B}}
$$

where $P_{1}=P_{2}=100$ and $P_{3}=1000$, the center frequencies are $F_{1}=1000 \mathrm{~Hz}, F_{2}=500$ $\mathrm{Hz}$, and $F_{3}=-250 \mathrm{~Hz}, B=10^{4}$ with $W=5000$ and $T=1 \mu \mathrm{s}$. In particular, Figure 3 (Top) shows that the colored noise is asymmetric about $f=0$ and multimodal. Hence, the spectrum of the optimal transmit signal is more complex and interesting. Here we see effects predicted by $(2.9)$ for the first time. In particular, when $P_{N}(f)$ falls below $1 / \lambda=14.3=11.55$ dB the optimal spectrum shows significant spectral content.

In contrast to the ROC curves in Example 1, the ROC curves for this example indicate a slight performance advantage for the optimal waveform over the LFM signal. Roughly speaking, the reason for the increased performance gap between the optimal design and the LFM signal is due to the increased complexity in the interference spectrum, which allows for the more complicated optimal design show a benefit when compared to the simplistic LFM spectrum. As we saw in the previous example, the LFM spectrum essentially assumes that everything in the environment responds the same over frequency. 

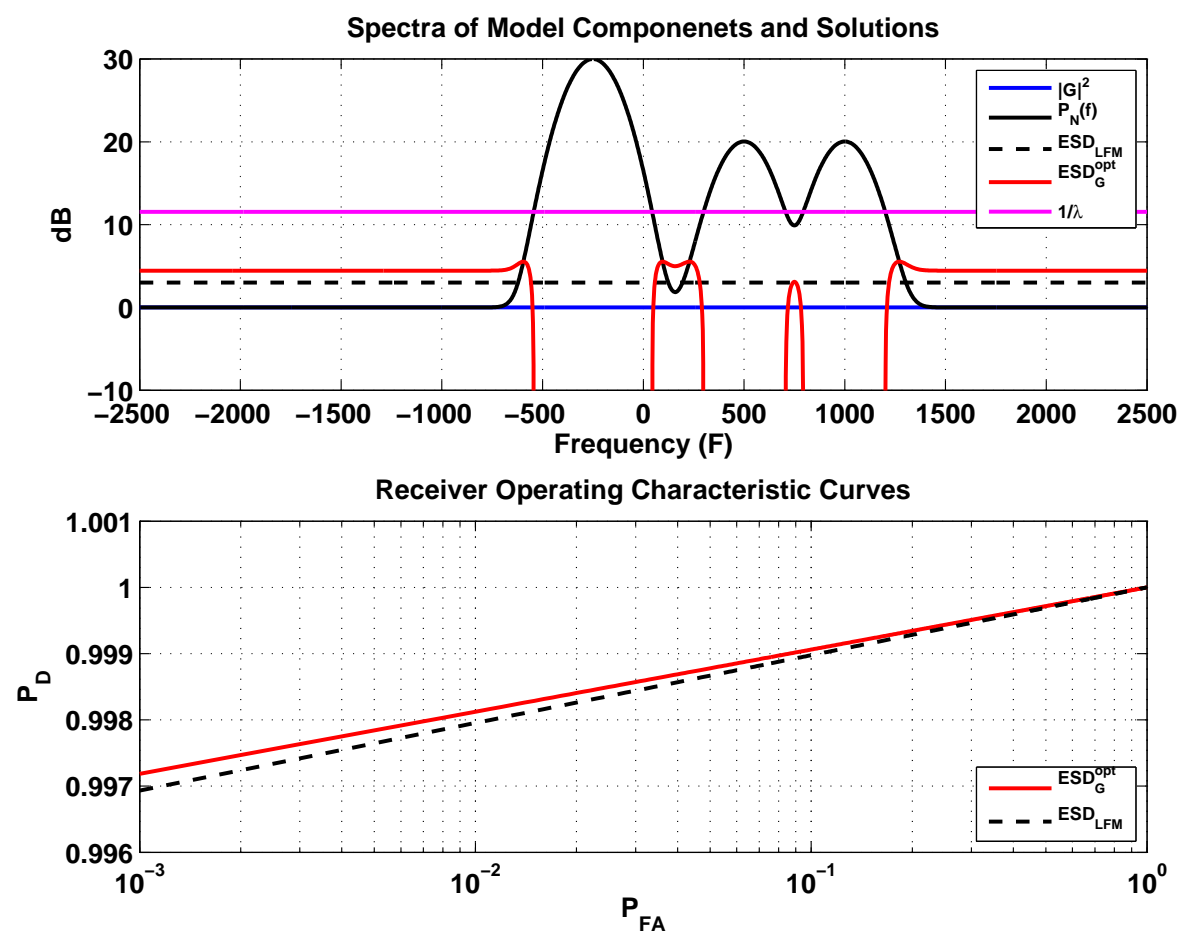

Figure 3: Example 2 - Point target in colored noise and white reverberation. (TOP) Spectrum of optimal signal (red), LFM (dashed black), Target response (solid blue), and Noise spectrum(solid black), (BOTTOM) ROC curves for optimal design (red) and LFM (black dashed) 


\subsubsection{Ex 3: Elastic Target in White Noise and White Reverberation}

In this and the following example we relax the point target assumption and consider an object that, when insonified by an acoustic pulse, has a response that is nonuniform over frequency. Fortunately, for simple geometric structures, such as a sphere or cylinder, one can reasonably model the response of the object to a given acoustic excitation as a Linear Time Invariant (LTI) system with frequency response predicted by the theory of resonance scattering (RST) $[22,23,24,25,26]$. For a spherical shell, equation (2.12) gives the mathematical from its frequency response,

$$
G(f)=\frac{1}{j 2 \pi f c} \sum_{i=0}^{\infty}(-1)^{n}(2 n+1) T_{n}(f)
$$

where $c$ is the speed of sound in water and for each index $i$ and frequency $f, T_{n}(f)$ is obtained by calculating the determinant of a matrix that depends of the specific size and material properties of the shell in question. For the shell considered in this thesis computing the sum in (2.12) up to $i=75$ (inclusive) is sufficient to resolve fine spectral detail in the magnitude spectrum of the shell.

Thus, determination of the backscatter from a given target is reduced to a simple convolution of the inverse Fourier transform of (2.12) with the transmit waveform, making it extremely convenient to analyze the performance of various waveform designs against a particular target. Specifically, we consider the spherical shell depicted in Figure 4 with the associated material properties summarized in Table 1.

The results for this example differ from the previous two in a few very interesting ways. First, Figure 5 (Top) indicates that the regions where the target responds most strongly are where the majority of signal energy is placed in the optimal design, which makes intuitive sense. In other words, the optimal design does not waste energy in regions of the spectrum where the target is unresponsive but rather saves its limited energy budget for spectral regions in the target response that exhibit strong resonances. 


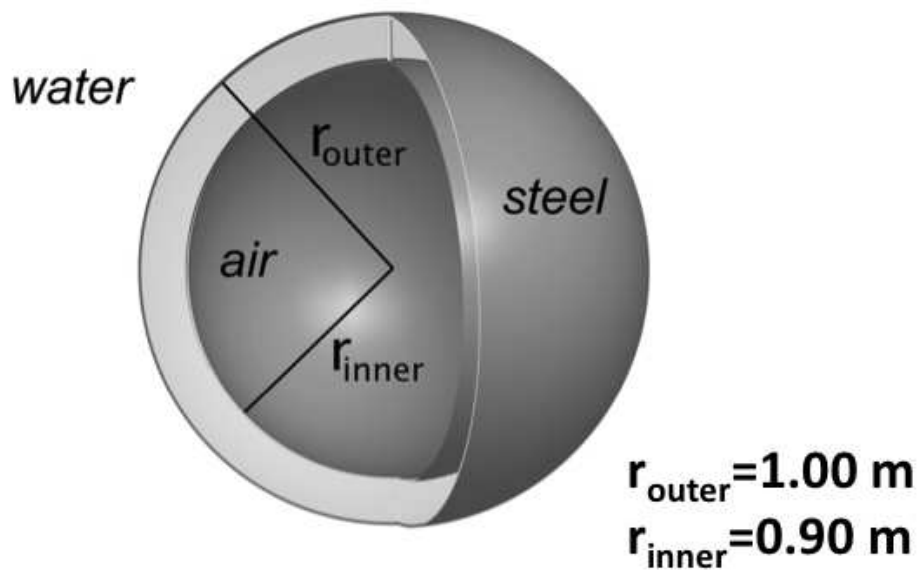

Figure 4: Diagram of Spherical Target used in Examples 3 and 4

Table 1: Material properties of the shell and surrounding environment.

\begin{tabular}{|c|c|c|}
\hline & $\begin{array}{c}\text { Density } \\
\left(\frac{\mathrm{kg}}{\mathrm{m}^{3}}\right)\end{array}$ & $\begin{array}{c}\text { Sound Speed } \\
\left(\frac{\mathrm{m}}{\mathrm{s}}\right)\end{array}$ \\
\hline Water layer & 1000 & 1500 \\
\hline Air & 1.0 & 340 \\
\hline Steel & 7800 & $\begin{array}{c}5880 \text { (dilatational) } \\
3140 \text { (shear) }\end{array}$ \\
\hline
\end{tabular}


The second major difference we observe is in the ROC curves shown in Figure 5 (Bottom). Here it is quite clear that the optimal design affords a significant performance gain over both the LFM signal and the optimal signal under the point target assumption. The reason for the performance gain is due to the complex nature of the target response. Intuitively, this makes sense in the context of an environment where reverberation is prevalent since a complex target leads to complex reflections that are specific to the target of interest and likely differ substantially from the spectrum of the transmitted signal. In other words, a complicated response from a given target (much like a finger print) helps in the decision making process. Finally, Figure 6 shows how (2.9) comes into play for this more complicated scenario. Again, we see that in spectral regions where ratio of noise power to target response falls below the $1 / \lambda$ threshold, energy is placed in these bands.

\subsubsection{Ex 4: Elastic Target in Colored Noise and White Reverberation}

In the final example of the chapter we make a slight modification to the parameters of Example 3. In particular, rather than a flat noise spectrum $P_{n}(f)$ we consider a colored noise spectrum, whose power grows linearly with frequency with $P_{n}(f)=1000|f|$ for $|f| \leq$ 1790.65. The results, shown in Figure 7, indicated that the optimal spectrum tends to concentrate more of its energy into the lower frequency resonances since the noise power is smallest in this portion of the spectrum.

In this chapter we saw how an optimal waveform can be derived to improve detection performance in a reverberant environment. One of the most interesting aspects of the solution is that it is expressed only in terms of a magnitude spectrum. Thus, in the following chapter we use spectral phase as a design parameter and derive time domain signals with optimal duration properties. 

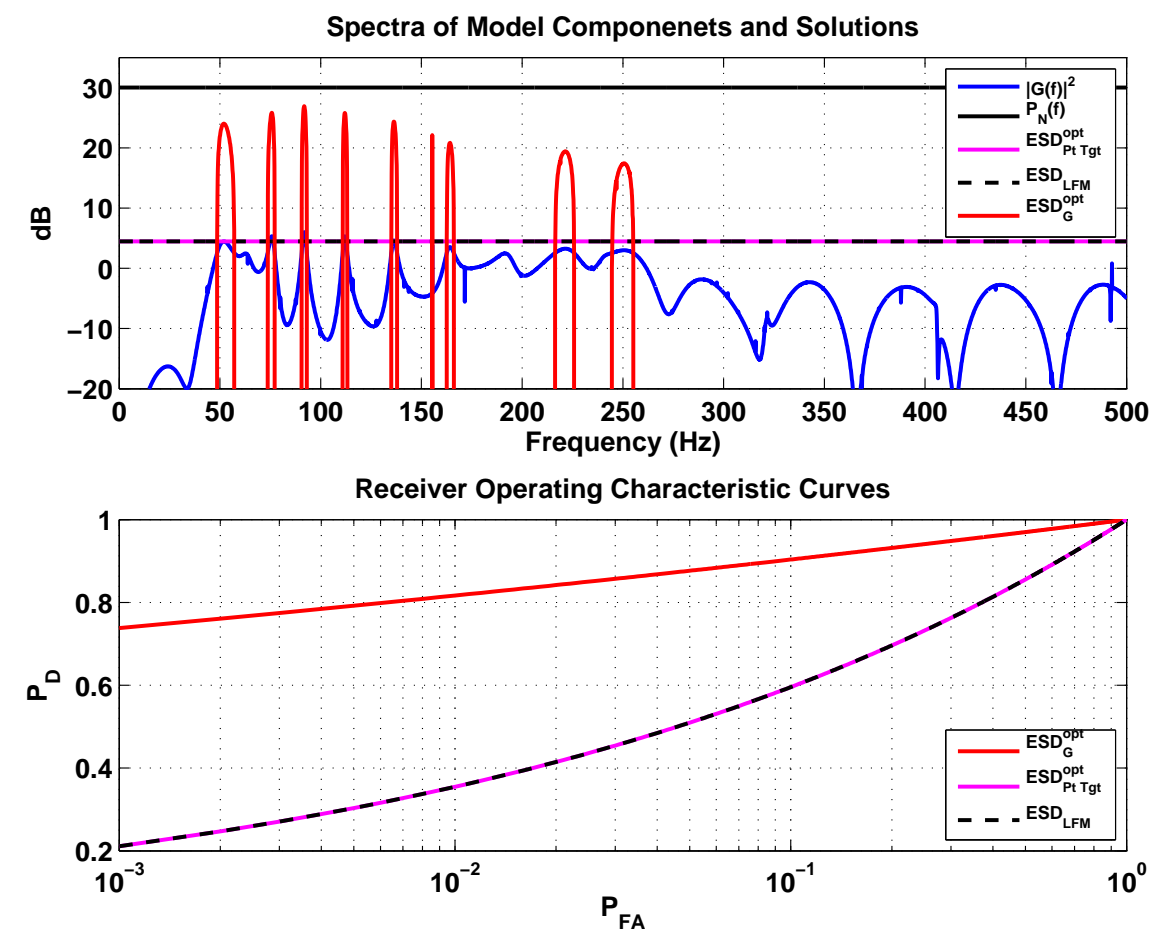

Figure 5: Example 3 - Elastic target in white noise and white reverberation. (TOP) Spectrum of optimal signal (red), LFM (dashed black), Spectrum of optimal signal under point target assumption, Target response (solid blue), and Noise spectrum (solid black), (BOTTOM) ROC curves for optimal design based on RST model of the sphere (red), optimal design based on point target assumption (magenta), and LFM signal(black dashed) 


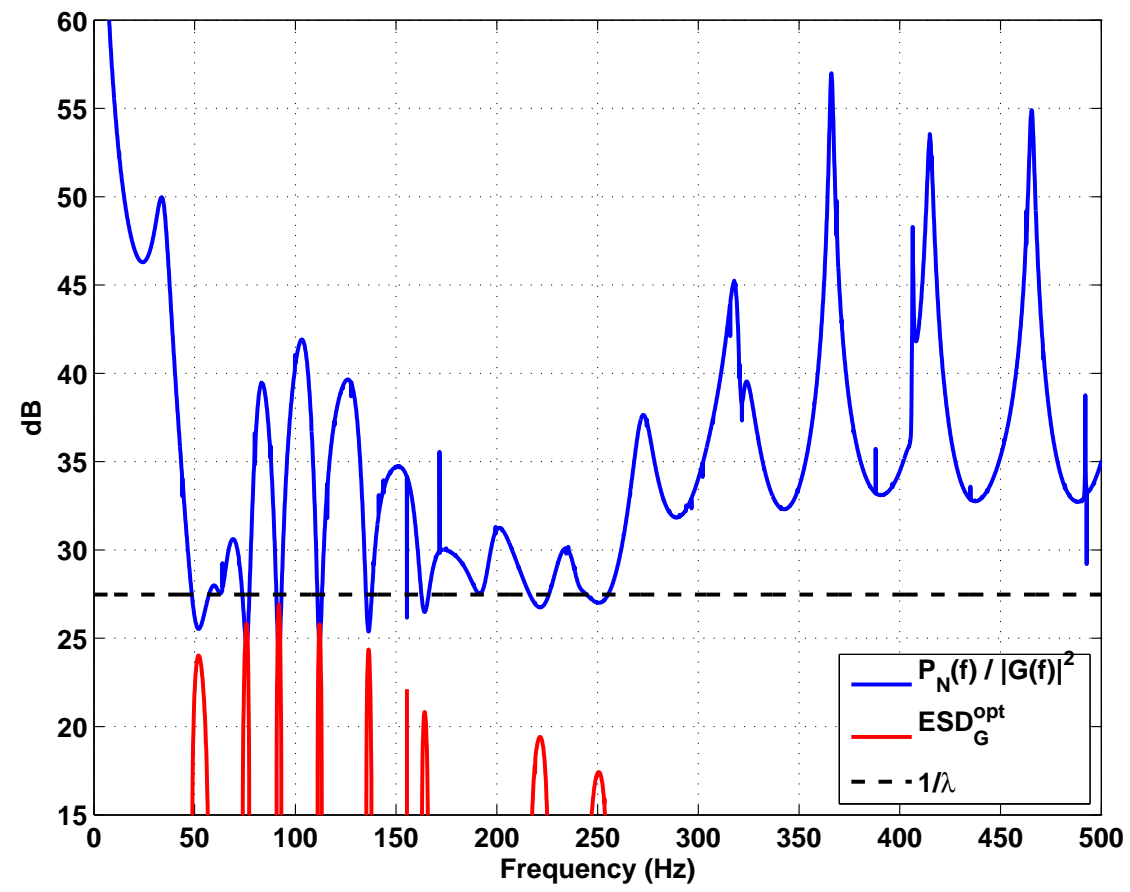

Figure 6: Example 3 - Relationship between noise, target, and optimal ESD. Threshold (Black Dashed) Optimal Spectrum (Red) and Noise Power to Target Response Ratio (Blue). Spectral regions where the blue curve exceeds the threshold receive energy. 

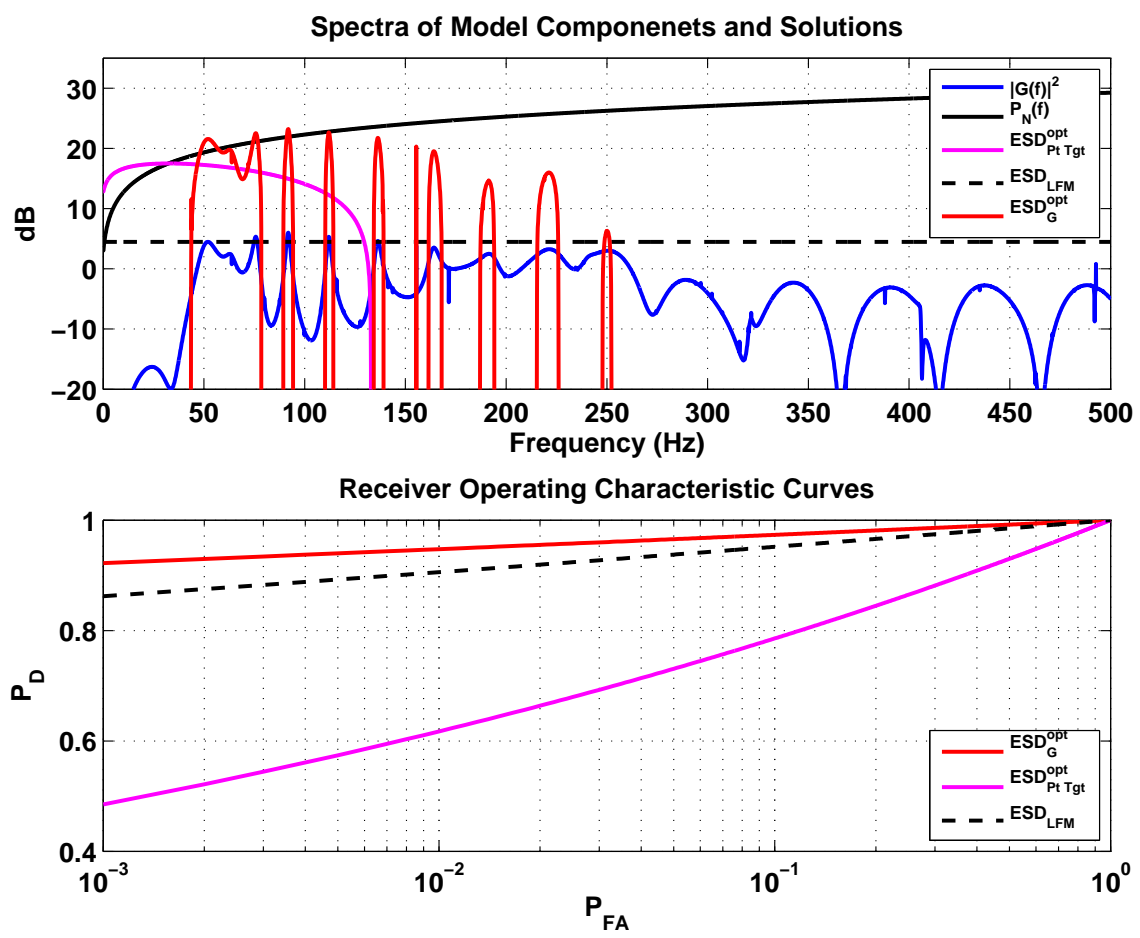

Figure 7: Example 4 - Elastic target in colored noise and white reverberation. (TOP) Spectra of Optimal Signal (red), LFM (magenta), Target (solid blue), and Noise (solid black), (BOTTOM) ROC curves for optimal design based on RST model of the sphere (red), optimal design based on point target assumption (magenta), and LFM (black dashed) 


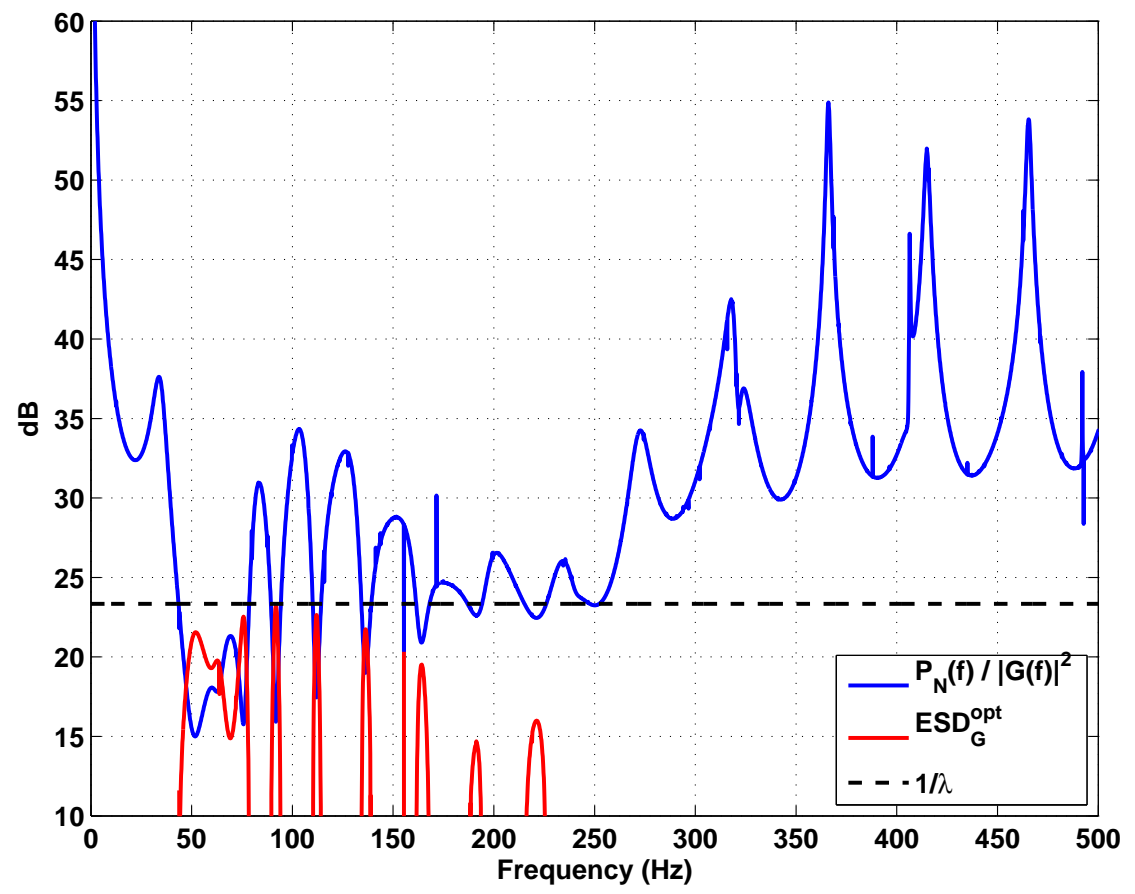

Figure 8: Example 4 - Relationship between noise, target, and optimal ESD. Threshold (Black Dashed) Optimal Spectrum (Red) and Noise Power to Target Response Ratio (Blue). Spectral regions where the blue curve exceeds the threshold receive energy. 


\subsection{OPTIMIZING SPECTRAL PHASE}

\subsection{INTRODUCTION}

The rate at which a sonar system probes the surrounding environment will impact the speed with which decisions are made. Accordingly, optimal waveforms with short duration are desirable since they can be transmitted more frequently, facilitating a prompt decision. Furthermore, signals with short durations are well known to be desirable for achieving high range resolution when the sonar task is localization. So, a case can made that one could use such signals to simultaneously perform detection and active ranging.

In the previous chapter we found that the waveform that maximizes the probability of detecting a target in reverberation only depends on the spectral magnitude of the transmit waveform and is uniquely determined by (2.8). Even though the spectral magnitude is unique, the time domain signal that has this spectral characteristic is not. In fact, there are an infinity of time domain signals that achieve a given spectral magnitude [27, pg. 788]. Since spectral phase is then essentially a function that we can do with as we please, it is natural to ask: Does spectral phase relate to any sonar parameters of interest?

The answer to this question is yes and the parameter of interest is duration. It is this link, the link between temporal duration and spectral phase, that we explore in this chapter. 


\subsection{THE OPTIMAL SPECTRAL PHASE}

For the purposes of this chapter it convenient to define Fourier Transform pairs by

$$
\begin{aligned}
s(t) & =\frac{1}{\sqrt{2 \pi}} \int S(\omega) e^{j \omega t} d \omega \\
S(\omega) & =\frac{1}{\sqrt{2 \pi}} \int s(t) e^{-j \omega t} d t
\end{aligned}
$$

It will also be convenient to express the spectrum $S(\omega)$ in terms of its amplitude $B(\omega)$ and phase $\psi(\omega)$ in the following form

$$
S(\omega)=B(\omega) e^{j \psi(\omega)}
$$

Duration, defined in terms of the temporal standard deviation (square root of the variance)

$$
\sigma_{t}^{2}=\frac{1}{E} \int(t-\langle t\rangle)^{2}|s(t)|^{2} d t
$$

with

$$
\langle t\rangle=\frac{1}{E} \int t|s(t)|^{2} d t
$$

and can be equivalently expressed in terms of the spectral magnitude, $B(\omega)$, and spectral phase, $\psi(\omega)$, as $[28]$

$$
\sigma_{t}^{2}=\int B^{\prime 2}(\omega) d \omega+\int\left(\psi^{\prime}(\omega)+\langle t\rangle\right)^{2} B^{2}(\omega) d \omega
$$

where ' denotes the derivative of the function. Also, henceforth we assume that $\int B^{2}(\omega) d \omega=$ $E=1$ for mathematical convenience.

In the remainder of this chapter we derive the spectral phase $\psi(\omega)$ that minimizes duration, maximizes duration, and blends these two extremes while constraining the time domain signal to have a specified magnitude spectrum. 


\subsubsection{The Minimum Duration Signal}

Since the optimal transmit waveform fixes the spectral magnitude, then in order to minimize the duration, we see from (3.6) that we need to choose the spectral phase so that the second integral is zero, by which we have the solution,

$$
-\psi_{\min }^{\prime}(\omega)=\langle t\rangle=t_{0}
$$

or, in other words, we need to select the signal so that it has constant group delay. Accordingly, the time domain signal with minimum duration and optimal spectral magnitude $B_{\text {opt }}(\omega)$ is given by,

$$
s_{\text {min-dur }}(t)=\frac{1}{\sqrt{2 \pi}} \int B_{o p t}(\omega) e^{j \omega\left(t-t_{o}\right)} d \omega
$$

and the minimum duration that is achieved is

$$
\sigma_{t-\min }^{2}=\int B_{o p t}^{\prime 2}(\omega) d \omega
$$

The practical utility of this result lies in the fact that signals with short duration can be transmitted more frequently and allow for high range resolution. The trade-off, however, is that the peak time domain energy, defined as $\max \left\{|s(t)|^{2}\right\}$, will generally be higher than an optimal waveform with the same spectral magnitude but longer duration. Intuitively, this can be understood by recalling that Parseval's Theorem states that the energy computed in the time domain is the same as that computed in the frequency domain. Hence, if the energy remains fixed in the frequency domain, as it will since $B(\omega)$ is fixed, a signal with this spectral magnitude, compressed in time, must have larger peak values. Thus, since the peak energy may be a limiting factor for the transmitter, we next consider the maximum duration solution, and then a combination that allows a blending between the two. 


\subsubsection{The Maximum Duration Signal}

Returning to (3.6) and recalling that $B(\omega)$ is already determined by the optimal detection criterion of the previous section, our aim is to maximize the duration by solving

$$
\max _{\psi^{\prime}(\omega)} \int\left(\psi^{\prime}(\omega)+\langle t\rangle\right)^{2} B^{2}(\omega) d \omega
$$

For square-integrable functions (which we will take to be the case), this integral is maximized when [29]

$$
\left(\psi^{\prime}(\omega)+\langle t\rangle\right)^{2} \sim B^{2}(\omega)
$$

by which we obtain

$$
\psi_{\max }^{\prime}(\omega)=K B_{o p t}(\omega)-t_{0}
$$

and therefore the spectral phase is

$$
\psi_{\max }(\omega)=K \int_{-\infty}^{\omega} B_{\text {opt }}(w) d w-t_{0} \omega
$$

where $K$ is an arbitrary real constant. Thus, the optimal maximum duration signal is

$$
s_{\max -\mathrm{dur}}(t)=\frac{1}{\sqrt{2 \pi}} \int B_{\text {opt }}(\omega) e^{j\left(\psi_{\max }(\omega)+\omega t\right)} d \omega
$$

with duration

$$
\sigma_{t, \max }^{2}=\int B_{o p t}^{\prime 2}(\omega) d \omega+K^{2} \int B_{o p t}^{4}(\omega) d \omega
$$

Thus, we see that we can make (3.14) arbitrarily large by allowing $K$ to grow without bound. Although true in theory, practical limitations will restrict our ability to produce such a signal. Furthermore, as we will see in section 3.3 a discrete time implementation will exhibit temporal aliasing if $K$ does not satisfy

$$
|K| \leq \frac{T-t_{0}}{\max \left(B_{\text {opt }}(\omega)\right)}
$$

This constraint on $K$ is justified by virtue of the fact that $-\psi^{\prime}(\omega)$ corresponds to the group delay which, for narrowband signals, corresponds to the amount of time delay at that frequency. Therefore, in a discrete implementation, $K$ must be chosen such that the spectral component that is delayed the most does not exceed the time interval, $T$, of the signal, if temporal aliasing is to be avoided. 


\subsubsection{Blending the Maximum and Minimum Duration Solutions}

The maximum duration signal will have lower peak power relative to the minimum duration signal, but so, too, will the transmission rate be lower. A compromise can be obtained by considering a blending of the two temporal designs presented in the previous sections. Namely, consider a linear combination of the minimum and maximum duration optimal signals, with spectral phase given by

$$
\psi_{\text {blend }}(\omega)=\alpha\left(K \int_{-\infty}^{\omega} B^{2}(\gamma) d \gamma\right)-t_{0} \omega
$$

where $0 \leq \alpha \leq 1$. In this way we can smoothly transition between the maximum $(\alpha=1)$ and the minimum duration $(\alpha=0)$ signal designs. 
Table 2: Duration and Peak-to-Average Energy Results

\begin{tabular}{|c|c|c|}
\hline & $\sigma_{t}(\mathrm{sec})$ & $E_{p k-t o t}$ \\
\hline Min & $2.22 \times 10^{-2}$ & 1185.44 \\
\hline Min Phase & $6.55 \times 10^{-2}$ & 199.76 \\
\hline Blend & $8.42 \times 10^{-2}$ & 46.33 \\
\hline Max & $16.6 \times 10^{-2}$ & 33.66 \\
\hline
\end{tabular}

\subsection{EXAMPLES}

In this section, we summarize numerical results for various waveforms all with the same optimal magnitude spectrum (obtained from Example 4 in Chapter 2), but with spectral phases ranging from the minimum to the maximum duration solutions presented in section 3.2. The duration and peak to total energy for each of the three cases are summarized in Table 2. Also, for the purposes of comparison, we include the minimum phase signal design which, for a given magnitude spectrum, is the impulse response of a system having all of its poles and zeros inside the unit circle. The minimum phase signal design, implemented based on MatLab code given in [30], is relevant since it is well known that minimum phase systems have impulse responses that maximally concentrate their energy in the lowest samples, a matter elaborated on in Appendix A.

Figure 9 (main panel) shows spectrograms (with 20dB dynamic range) for each of the three cases treated in this chapter and the minimum phase design just introduced, along with the associated temporal (lower panel) and spectral (left panel) representations. As expected, Figure 9 (a) shows that for the minimum duration solution the signal has its energy concentrated in the most narrow time interval but has a significant peak energy located around its temporal average. Figure 9 (b) shows a spectrogram of the maximum duration signal which has its energy spread out over time, resulting in a significant increase in duration and a corresponding decrease in peak-to-total energy. In Figure 9 (c) we show 
a blended signal, obtained via (3.16) with $\alpha=0.5$. For this signal, the duration and the peak-to-total energy are seen to lie within the extremes resulting from the aforementioned two cases. Finally, in Figure $9(\mathrm{~d})^{1}$ we take note that while the minimum phase signal tends to bunch its energy in the lower samples (i.e. those to the right of $t=0$ ) it is still broader in terms of duration as a measure of its temporal energy spread, an observation confirmed numerically in Table 2 .

\footnotetext{
${ }^{1}$ For the sake of uniformity with Figures 9 (a) - Figure 9 (c) we have delayed the minimum phase signal design shown in Figure 9 (d) by 2 seconds, an operation that does not change duration. In contrast to the minimum duration signal design discussed in this chapter, there is nothing inherent in the minimum phase signal design that produces such a delay. With reference to Figure 9 (d), one must think of all samples to the right of $t=2$ as having the minimum-delay property, a property expounded on further in Appendix A.
} 

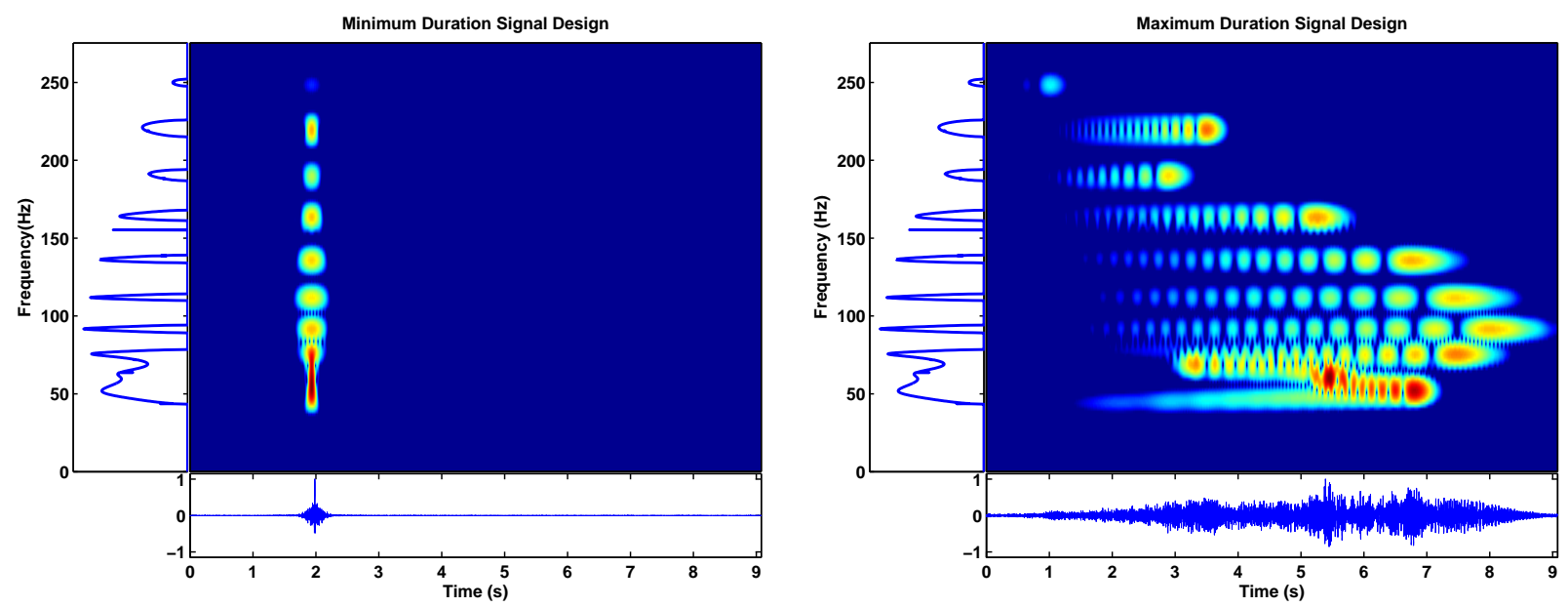

(a) Minimum duration solution yielding narrow pulse (b) Maximum duration solution yielding a broad pulse width and high peak energy width and low peak energy. Here $K$ was chosen to be just above the lower bound given in (3.15) with $t_{0}=0$

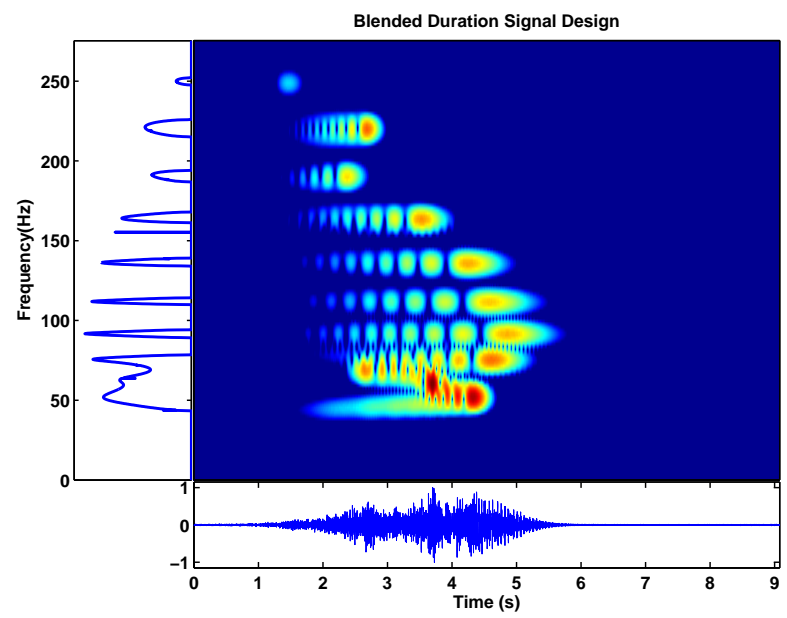

(c) Blended duration solution for $\alpha=0.5$ which balances narrow pulse width and low peak energy

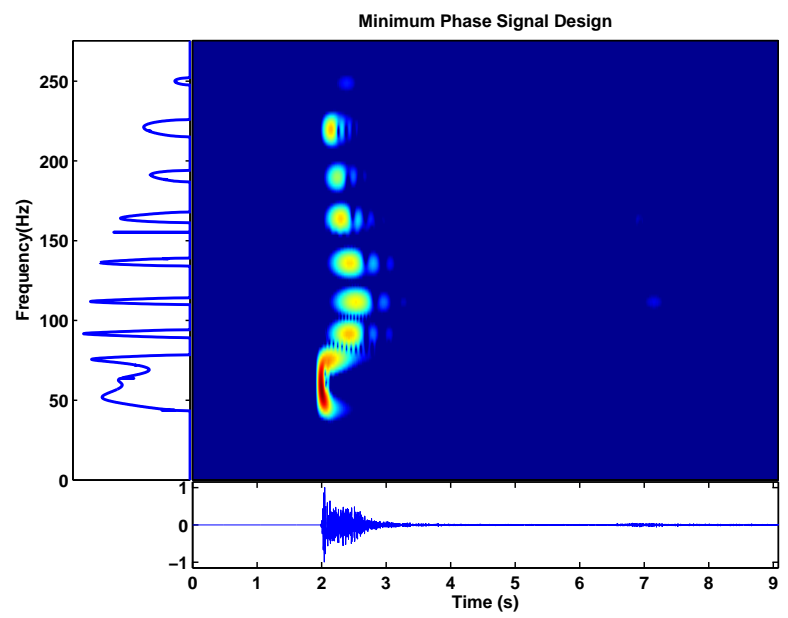

(d) Minimum phase solution

Figure 9: Examples - Spectrograms (20dB dynamic range) showing time-frequency properties of each spectral phase based signal design 


\subsection{OPTIMIZING TEMPORAL CONCENTRATION}

\subsection{INTRODUCTION}

In Chapter 2 we studied the problem of signal design for maximizing the probability of detecting an elastic target in environments where reverberation and ambient noise are present. We found that the waveform that maximizes the probability of detection is specified only in terms of its spectral magnitude. This observation was exploited in Chapter 3 to design a signal with specific time-domain duration properties. In particular, for a fixed spectral magnitude we derived the optimal spectral phase function that minimizes or maximizes the duration. Blending these two extremes allows for a trade-off between peak power and duration.

This chapter is concerned with deriving signals that optimize detection while maximally concentrating the signal energy within a finite discrete-time interval. The solution necessitates a trade-off between concentration and detection performance. The problem formulation allows indirect control over the degree of performance loss through a scalar design parameter, $\epsilon$.

We begin by outlining the theory originally set forth by Slepian, Pollak, and Landau $[14,15,16,17]$ related to the so-called concentration problem, which itself was motivated by the fundamental limit known as the duration-bandwidth product (a.k.a the uncertainty principle). Of particular interest to the main results of this chapter is the discrete-time continuous frequency concentration problem [31, pp. 101-109], which is concerned with finding the sequence $s(n)$ whose energy is maximally concentrated in the index range $(0, N-$ $1)$ and is band-limited to $(-W, W)$ with $|W|<\pi$. We modify the classical formulation so that the spectrum of the optimal $s(n)$ is not only maximally concentrated in $(0, N-1)$ and 
band-limited to $(-W, W)$ but is also (1) spectrally similar to a given magnitude spectrum (such as $\left.B_{\text {opt }}\right)$, (2) nonnegative, and (3) symmetric in $(-W, W)$. The problem is subsequently recast as a constrained nonlinear program with constraints reflecting (1), (2), and (3). We conclude the theoretical developments of this chapter by connecting the solution to this modified formulation to the minimum duration solution from Chapter 3. 


\subsection{THE CLASSICAL SLEPIAN PROBLEM}

The theory that our considerations are based on is due to the work of Slepian, Pollak, and Landau who, beginning in 1961, wrote a series of papers $[14,15,16,17]$ that studied various aspects of what they termed the concentration problem. This problem takes on two dual forms; the duality arises depending on whether the goal is concentration in time or concentration in frequency.

The most popular form of the discrete-time/continuous frequency concentration problem seeks to answer the following question: What signal, limited to the discrete-time range $(0, N-$ $1)$, is maximally concentrated in the frequency interval $(-W, W)$ ? This is an interesting question because as Slepian [32] and others [28,33] have noted, there is a fundamental limit

on the extent to which a signal can be localized, simultaneously, in time and frequency. The limit is known as the duration-bandwidth product theorem in the field of signal processing or the uncertainty principle in physics. Specifically, the theorem states that the product of the duration and bandwidth of a signal $s(t)$ is always larger than a fixed non-zero constant. Specifically, if we define duration $\sigma_{t}$ via $^{1}$

$$
\sigma_{t}^{2}=\int(t-\langle t\rangle)^{2}|s(t)|^{2} d t, \quad\langle t\rangle=\int t|s(t)|^{2} d t
$$

and bandwidth $\sigma_{\omega}$ via

$$
\sigma_{\omega}^{2}=\int(\omega-\langle\omega\rangle)^{2}|S(\omega)|^{2} d \omega, \quad\langle\omega\rangle=\int \omega|S(\omega)|^{2} d \omega
$$

then the duration-bandwidth product theorem states that

$$
\sigma_{t} \sigma_{\omega} \geq \frac{1}{\sqrt{2}}
$$

\footnotetext{
${ }^{1}$ Recall, we assume $\int|s(t)|^{2} d t=\int B^{2}(\omega) d \omega=E=1$
} 
Mathematically, Slepian et.al. reformulated the idea of time-frequency localization by introducing the notion of concentration in frequency (or concentration in time in its dual form). To do so in the context of discrete time and continuous frequency, the Discrete-Time Fourier Transform (DTFT) is needed and is defined as follows

$$
\begin{array}{ll}
(\text { DTFT }) & S(\omega)=\sum_{n=-\infty}^{\infty} s(n) e^{-j \omega n} \quad \omega \in[-\pi, \pi] \\
(\text { IDTFT }) & s(n)=\frac{1}{2 \pi} \int_{-\pi}^{\pi} S(\omega) e^{-j \omega n} d \omega
\end{array}
$$

where it is again convenient to represent the spectrum in terms its amplitude $B(\omega)$ and phase $\psi(\omega)$

$$
S(\omega)=B(\omega) e^{j \psi(\omega)}
$$

The objective is then one of finding $s(n)$ that maximizes

$$
\beta^{2}(W)=\frac{\int_{-W}^{W}|B(\omega)|^{2} d \omega}{\int_{-\pi}^{\pi}|B(\omega)|^{2} d \omega} \quad W \in[-\pi, \pi]
$$

The signals that maximize this ratio are members of the family of functions known as prolate spheroidal wave functions. Once it was known that these functions solved the problem as stated, they found their way into some important practical applications. One of the more notable applications is in the Thomson multitaper method $[34,31]$ that appears in the theory of spectral estimation.

A dual form of this problem can also be posed. In particular, suppose we wish to find the signal that is band limited to $(-W, W)$ and is maximally concentrated in a predefined index range $(0, \mathrm{~N}-1)$. Similar to $(4.7)$, the discrete-time concentration measure is defined as

$$
\alpha^{2}(N)=\frac{\sum_{n=0}^{N-1}|s(n)|^{2}}{\sum_{n=-\infty}^{n=\infty}|s(n)|^{2}}
$$

where the goal is again to find $s(n)$, but this time it is to maximize $\alpha^{2}(N)$. It is this formulation that we consider throughout the remainder of this chapter. 


\subsection{THE MODIFIED SLEPIAN CONCENTRATION PROBLEM}

The concentration in time problem as posed at the end of the previous section places no restriction on the spectrum of the desired signal, other than it is to be band-limited. In this section we propose a modification to the classical formulation that imposes constraints on the spectral shape of the signal that maximizes equation (4.8). In particular, we address the question: What real discrete-time signal, $s(n)$, band-limited to $(-W, W)$, is maximally concentrated in the index range $(0, N-1)$ while simultaneously making $S(\omega)$ as close as possible to a given spectrum.

\subsubsection{Mathematical Formulation: Discrete Time/Continuous Frequency}

Using (4.4) and (4.5) we can express (4.8) in the frequency domain as

$$
\alpha^{2}(N)=\frac{\int_{-W}^{W} \int_{-W}^{W} H(\omega) N D_{N}\left(\omega-\omega^{\prime}\right) H^{*}\left(\omega^{\prime}\right) d \omega d \omega^{\prime}}{\int_{-\pi}^{\pi}|H(\omega)|^{2} d \omega}
$$

where

$$
D_{N}=\frac{\sin \left(\frac{N}{2}\left(\omega-\omega^{\prime}\right)\right)}{\sin \left(\frac{1}{2}\left(\omega-\omega^{\prime}\right)\right)}
$$

and

$$
H(\omega)=S(\omega) e^{j \frac{\omega(N-1)}{2}}
$$

The ratio of integrals in (4.9) can be shown [35] to take on its maximum value when the following integral equation is satisfied

$$
\int_{-W}^{W} N D_{N}\left(\omega-\omega^{\prime}\right) H(\omega) d \omega=\alpha^{2}(N) H\left(\omega^{\prime}\right)
$$

This equation is a continuous form of the ubiquitous eigenvalue problem. 


\subsubsection{Mathematical Formulation: Discrete Time/Discrete Frequency}

Though convenient for theoretical analysis, the DTFT leads to a solution (i.e. (4.12)) in terms of continuous variables which cannot be exactly implemented on a digital computer. Therefore, we discretize (4.12) and recast it in the following vector matrix form of the eigenvalue problem

$$
A H=\alpha^{2}(N) H
$$

where ${ }^{2}$

$$
A(p, q)=\frac{\sin \left(\frac{N}{2}\left(\omega_{p}-\omega_{q}\right)\right)}{\sin \left(\frac{1}{2}\left(\omega_{p}-\omega_{q}\right)\right)} \quad p, q=1, \ldots, M
$$

denotes the entries of the matrix $A$ with $A \sim M \times M$ and $H \sim M \times 1$ is given as

$$
H=\left[\begin{array}{llll}
H\left(\omega_{1}\right) & H\left(\omega_{2}\right) & \cdots & H\left(\omega_{M}\right)
\end{array}\right]^{T}
$$

where $\omega_{p}$ and $\omega_{q}$ are discrete frequencies.

\subsubsection{Mathematical Formulation: Nonlinear Program}

In this section we develop a nonlinear programming approach to solve the modified concentration problem. However, before we incorporate the modifications, we first introduce a simpler nonlinear programming formulation to which the modifications are made. In general, one can show that the solution to the following constrained nonlinear program

$$
\begin{aligned}
& \max _{H} H^{T} A H \\
& \text { st. } H^{T} H=1
\end{aligned}
$$

is the eigenvector of $A$ corresponding to its maximum eigenvalue [36, pp. 224-225]. It is to this formulation that we add three constraints.

\footnotetext{
${ }^{2}$ Here we assume that $\mathrm{M}$ is even
} 
The first constraint is the spectral similarity constraint,

$$
\left\|H-B_{\text {opt }}\right\|^{2} \leq \epsilon
$$

which enforces our desire to generate a solution that is arbitrarily close (in the least squares sense) to a given spectrum, where $\|\cdot\|$ denotes the standard Euclidian norm. Here $B_{\text {opt }}$ is a $M \times 1$ vector of frequency domain samples of a desired magnitude spectrum. In the following we take these samples to be from the optimal spectrum given by (2.8). The parameter $\epsilon>0$ is a user specified scalar parameter that serves as a means to trade detection performance for duration. As we will see in the following subsection, as $\epsilon \rightarrow 0, P_{D}$ is maximized for a given $P_{F A}$. The second constraint we add serves to ensure that $H$ is a valid (i.e. nonnegative) magnitude spectrum. Specifically,

$$
H \geq 0
$$

The last constraint we add is imposed to achieve spectral symmetry, and consequently, leads to a real-valued time domain signal. The constraint is formulated as

$$
R H=0
$$

where

$$
R=\left[\begin{array}{ll}
R_{1} & R_{2}
\end{array}\right]
$$

with

$$
R_{1}=I_{\frac{M}{2} \times \frac{M}{2}} \quad R_{2}=\left[\begin{array}{ccccc}
0 & 0 & \cdots & 0 & -1 \\
0 & 0 & \cdots & -1 & 0 \\
\vdots & \vdots & . \cdot & \vdots & \vdots \\
0 & -1 & \cdots & 0 & 0 \\
-1 & 0 & \cdots & 0 & 0
\end{array}\right]
$$

where $I_{\frac{M}{2} \times \frac{M}{2}}$ denotes the $\frac{M}{2} \times \frac{M}{2}$ identity matrix, $R_{2}$ is the negative of the reversal matrix with size $\frac{M}{2} \times \frac{M}{2}$, and therefore $R$ has size $\frac{M}{2} \times M$. This constraint is essentially a set of $M / 2$ linear equations that force $H\left(\omega_{i}\right)=H\left(\omega_{M-i+1}\right)$ for each $i \in[1,2, \ldots, M]$. 
Thus, the constrained nonlinear program we solve to obtain the solution to the modified Slepian problem is

$$
\begin{aligned}
\max _{H} H^{T} A H \\
\text { subject to } H^{T} H=1 \\
\left\|H-B_{o p t}\right\|^{2} \leq \epsilon \\
H \geq 0 \\
R H=0
\end{aligned}
$$

The formulation of and the solution to the nonlinear program given in (4.22) is the first core result of this chapter; the following subsection presents the second.

\subsubsection{Connection to the Minimum Duration Solution}

In 3.2.1 we derived the spectral phase function $\psi(\omega)$ that minimizes (3.6) and found that choosing $\psi(\omega)$ to be a linear function of $\omega$ (c.f. (3.7)) corresponds to a time domain signal $s(t)$ that has minimum duration. In this chapter we considered the problem of obtaining an optimal signal that is maximally concentrated in time. In this subsection we show the connection between solving (4.22) and minimizing (3.6).

In the classical discrete-time concentration problem formulation of subsection 4.3.1, $H_{\text {opt }}(\omega)$ is first obtained by solving the continuous eigenvalue problem in $(4.12)$, then $s(n)$ is subsequently computed by performing an inverse DTFT. Solving (4.11) for $S(\omega)$ and substituting into (4.5) yields

$$
\begin{aligned}
s(n) & =\frac{1}{2 \pi} \int_{-\pi}^{\pi} H_{o p t}(\omega) e^{-j \frac{\omega(N-1)}{2}} e^{j \omega n} d \omega \\
& =\frac{1}{2 \pi} \int_{-\pi}^{\pi} S_{o p t}(\omega) e^{j \omega n} d \omega
\end{aligned}
$$

where $S_{\text {opt }}(\omega)=H_{\text {opt }}(\omega) e^{-j \frac{\omega(N-1)}{2}}$. By $(4.22)$ it is clear that for each $\omega$ as $\epsilon \rightarrow 0, H_{\text {opt }}(\omega) \rightarrow$ $B_{\text {opt }}(\omega)$ and hence $S_{\text {opt }}(\omega) \rightarrow B_{\text {opt }}(\omega) e^{j \psi(\omega)}$ with $\psi(\omega)=\frac{\omega(N-1)}{2}$, which is a linear function of frequency. Hence, as $\epsilon \rightarrow 0, S_{\text {opt }}(\omega)$ corresponds to a signal with constant group delay and (3.7) is satisfied with $t_{0}=\frac{(N-1)}{2}$. So, we have demonstrated that as $\epsilon \rightarrow 0, P_{D}$ is maximized for a given $P_{F A}$ since $\left|S_{\text {opt }}(\omega)\right| \rightarrow B_{\text {opt }}(\omega)$ for that case. 


\subsection{EXAMPLES}

In the previous sections of this chapter we described the theory behind the classical and modified Slepian problems. In this section we highlight the main points of the theory with four examples. In each example the problem in (4.22) must be solved. To do so we use the MatLab ${ }^{\circledR}$ Optimization Toolbox [37] to implement the method of sequential quadratic programing (SQP) [38].

\subsubsection{Ex 1: Equivalence of Classical and Modified Formulations as $\epsilon \rightarrow \infty$}
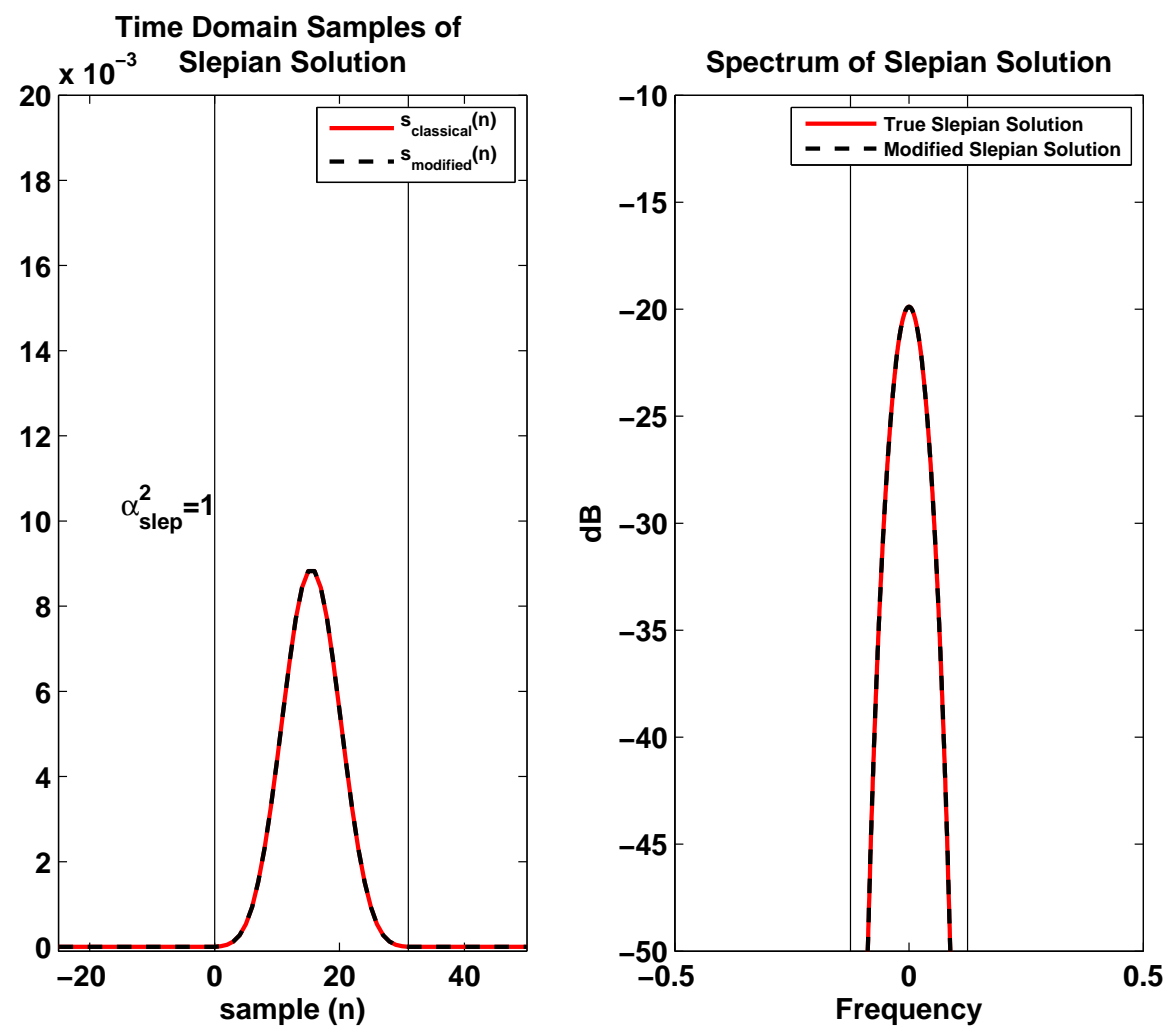

Figure 10: Example 1 - Equivalence of Classical and Modified Methods for $\epsilon \rightarrow \infty$. Solution to concentration in time problem by classical (solid red) and modified (dashed black) methods. 
The first example is meant illustrate the equivalence of the modified constrained concentration problem with the classical Slepian formulation, by taking $\epsilon$ to be sufficiently large. For $N=32$ and $W=1 / 8$, Figure 10 shows that both methods yield the same solution and exhibit the Gaussian shape, in both time (Left) and frequency (Right), that is characteristic of low order prolate spheroidal wave-functions resulting from small time-bandwidth products (i.e. $N W=4$ as in this example). For the sake of deriving the classical solution from the modified formulation we let $\epsilon=5$, a parameter value that is sufficiently large to ignore the spectral similarity constraint of (4.17). Of course, "large" depends on the magnitude of $B_{\text {opt }}(\omega)$ - i.e. if $B_{\text {opt }}(\omega)$ is on the order of $10^{6}$ vs. 10 , then $\left\|H-B_{\text {opt }}\right\| \sim 100$ is not large but it is large for $B_{\text {opt }}(\omega)$ on the order of 10 .

\subsubsection{Ex 2: Solution to Modified Formulation for $\epsilon=0.01$}

In the second example we incorporate the optimal spectrum from Example 4 in Chapter 3. In order to incorporate the optimal spectrum into (4.22) we normalized the frequency axis to $-0.5 \leq f \leq 0.5$, sampled the optimal spectrum at 388 equally spaced points in this interval, specified $W=0.25$, and $N=100$ samples. At this point it becomes clear why we chose the Discrete Time/Continuous Frequency formulation of the classical problem from the outset. This choice allows one to choose an arbitrary number of frequency domain samples of the optimal spectrum, which is not necessarily the same as the number of time domain samples to which the optimal signal is maximally concentrated within. In contrast, the classical Discrete Time/Discrete Frequency formulation requires an equal number of time and frequency domain samples to be specified. Having independent control over the number of time and frequency domain samples in the solution is an important property of the modified formulation since targets of interest in sonar applications exhibit sharp resonances that could easily be missed if frequency resolution was too coarse.

The upper left plot in Figure 11 shows both the solution to the modified problem and the minimum duration solution, as determined in 3.2.2. The fact that the two signals closely match one another supports the results from 4.3.4. However, the consequence of approaching the optimal solution from 3.2.2 is the existence of significant energy leakage outside of the first 

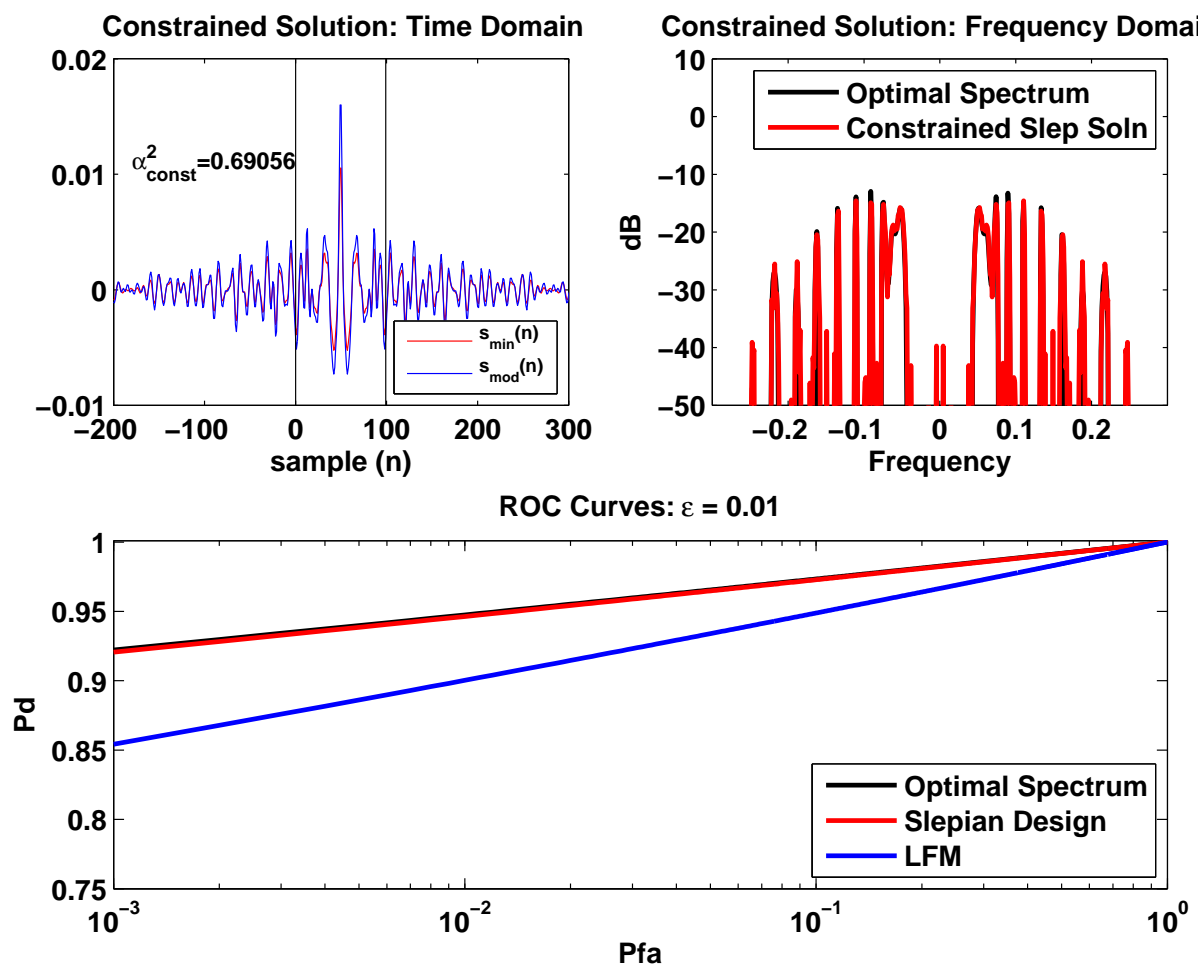

Figure 11: Example 2 - Signal Design Results for $\epsilon=0.01$. (UPPER LEFT) Time domain signal from modified formulation (blue) and minimum duration solution (red). (UPPER RIGHT) Optimal spectrum (Solid Black) and spectrum from modified solution (Solid Red). (BOTTOM) ROC curves for optimal spectrum (Solid Black), spectrum from modified solution (Solid Red) and LFM spectrum (Solid Blue). 
100 samples. This is because choosing $\epsilon=0.01$ effectively causes the temporal concentration aspect of the problem to be ignored and tends to match the optimal frequency spectrum very closely, as indicated in the upper right plot of Figure 11. As a result, and predicted in 4.3.4, the detection performance of the derived signal is nearly maximized as indicated by the ROC curves shown in the bottom plot of Figure 11. In general, the upper bound on the detection performance is given by that of the optimal spectrum from Chapter 2. Accordingly, as $\epsilon$ varies from small to large, the ROC curve associated with the modified solution falls further and further below that of the optimal signal.

\subsubsection{Ex 3: Solution to Modified Formulation for $\epsilon=0.3$}

In the third example all parameters associated with Example 2 remain intact except now we increase $\epsilon$ to 0.3 . The result is that the spectrum of the solution to the modified formulation (solid red in Upper Right plot of Figure 12) is not as closely matched to the optimal spectrum (solid black). The trade off between detection performance and duration is now clear since the energy is more concentrated within the first 100 samples while detection performance begins to fall away from the upper bound set by the optimal spectrum.

\subsubsection{Ex 4: Solution to Modified Formulation for $\epsilon=3.0$}

Finally, example 4 shows that allowing $\epsilon$ to get large leads to a solution that effectively ignores the optimal spectrum but is completely confined to the first 100 samples of the signal. Consequently, detection performance is so severely degraded that the LFM signal attains better performance in this case. 

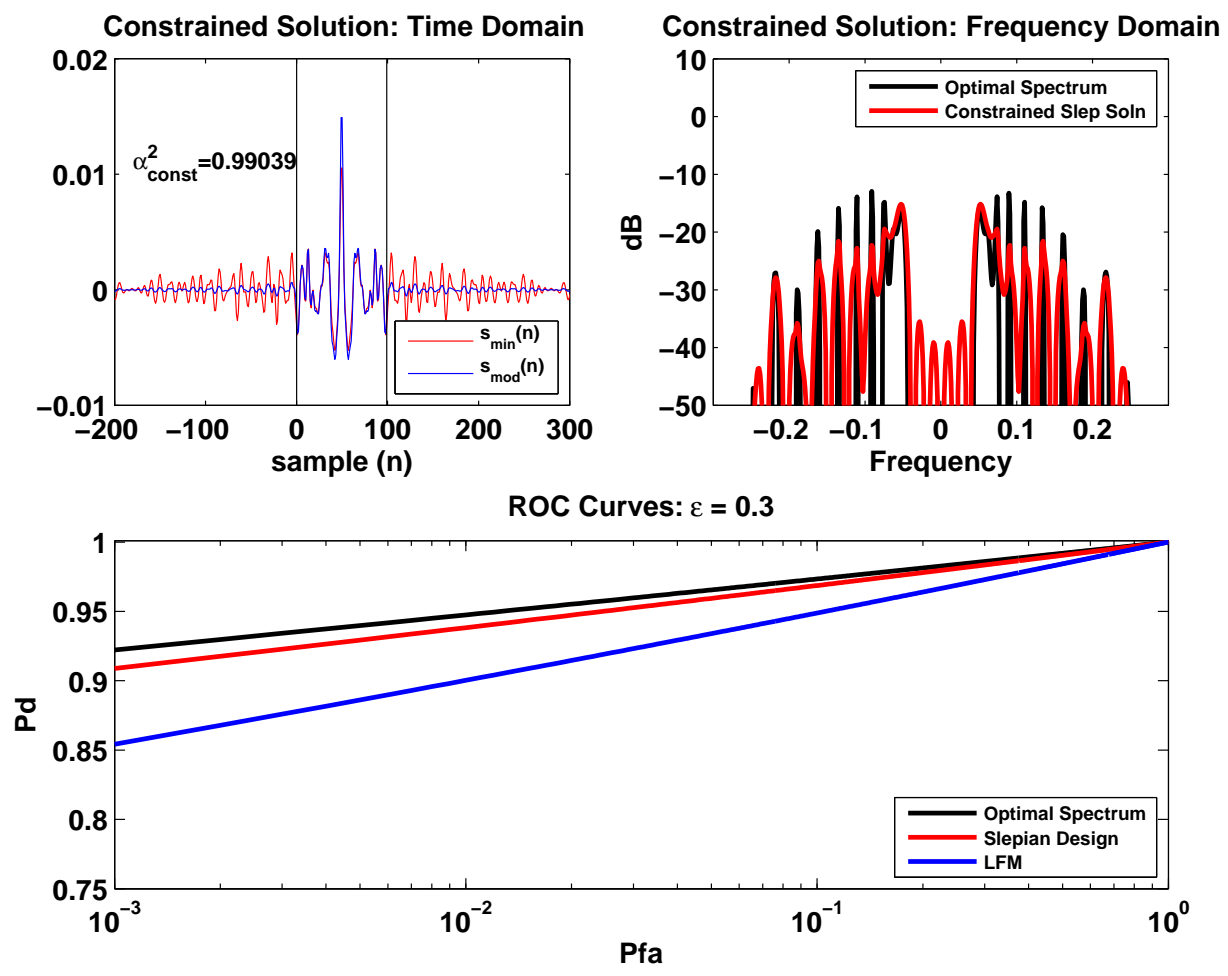

Figure 12: Example 3 - Signal Design Results for $\epsilon=0.3$. (UPPER LEFT) Time domain signal from modified formulation (blue) and minimum duration solution (red). (UPPER RIGHT) Optimal spectrum (Solid Black) and spectrum from modified solution (Solid Red). (BOTTOM) ROC curves for optimal spectrum (Solid Black), spectrum from modified solution (Solid Red) and LFM spectrum (Solid Blue). 

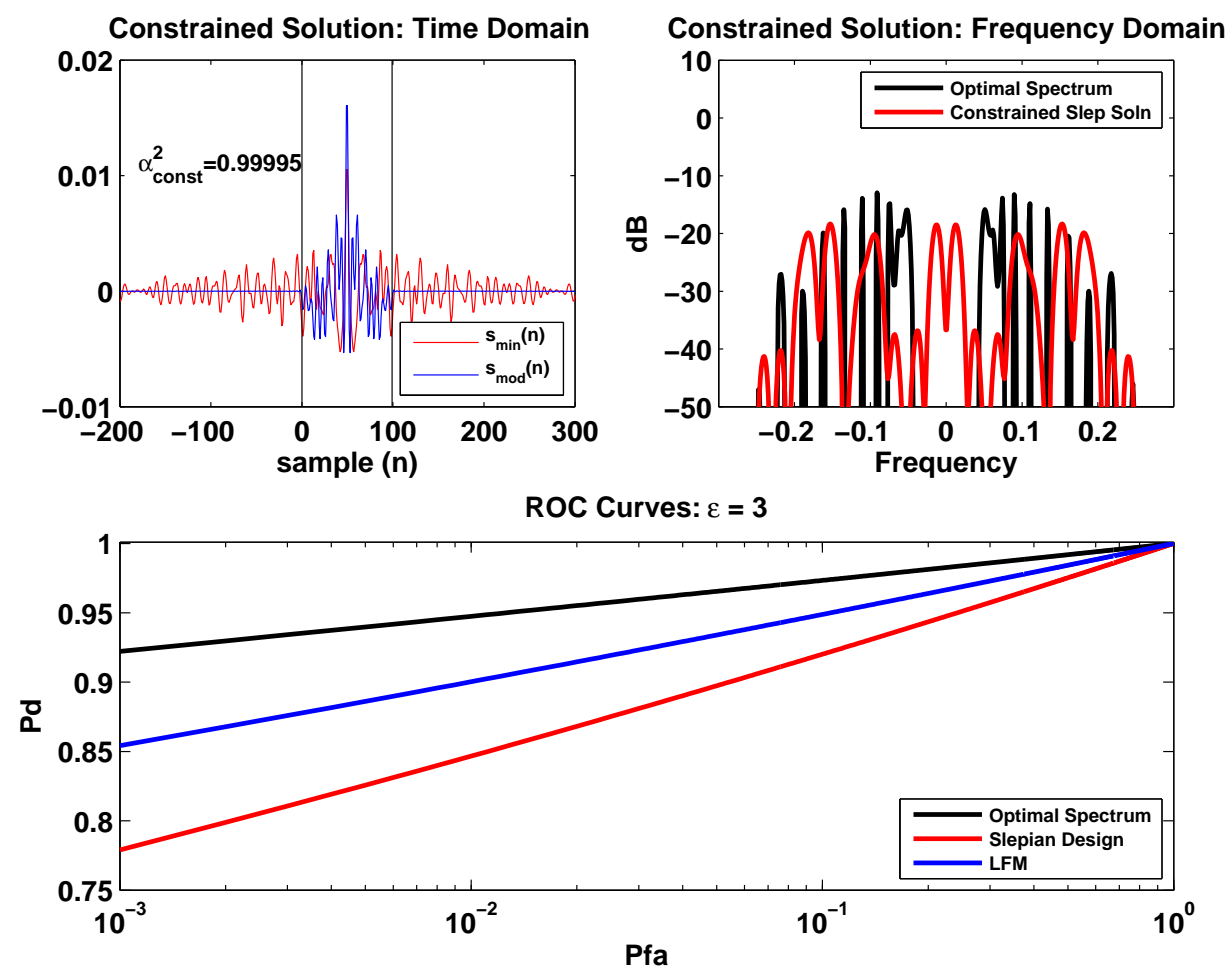

Figure 13: Example 4 - Signal Design Results for $\epsilon=3.0$. (UPPER LEFT) Time domain signal from modified formulation (blue) and minimum duration solution (red). (UPPER RIGHT) Optimal spectrum (Solid Black) and spectrum from modified solution (Solid Red). (BOTTOM) ROC curves for optimal spectrum (Solid Black), spectrum from modified solution (Solid Red) and LFM spectrum (Solid Blue). 


\subsection{CONCLUSIONS AND FUTURE DIRECTIONS}

In this work we considered two new signal design approaches that were based on the optimal spectral magnitude derived by Kay [4]. Each design exploited the fact that the magnitude spectrum of a signal does not uniquely define its time domain counterpart. In the first, we saw that by designing the spectral phase one can derive time domain signals with varying degrees of temporal duration while maintaining optimal spectral magnitude. We found that the trade-off for short duration signals was the presence of high peak energy (and vice versa). In the second approach we reformulated the problem in Chapter 4 to determine the time domain signal that is maximally concentrated in a predefined discrete-time range, with a spectral magnitude that is close (in the least-squares sense) to the optimal spectral magnitude. It was found that the more we concentrate in time, the greater the error in spectral magnitude, resulting in a loss in detection performance.

From a practical standpoint each of these design approaches has its place. Specifically, in situations where one needs to simultaneously test for the presence of a particular target and if present accurately estimate its range, the short duration signals of Chapters 3 and 4 should be considered. In contrast, if range resolution and short blanking times are less important than having lower peak power, the longer durations signals presented in Chapter 3 are favorable.

Some shortcomings of these and other existing approaches suggest some directions for further work. First, in each problem formulation we assumed a very specific target model. Rather than making such restrictive assumptions, it is desirable to design signals that are robust to target model variations $[39,40,41]$. This suggests the formulation of a signal design problem whereby the optimal solution is one that maximizes the minimum probability of detecting a target within an assumed class of potential target models. Along the same lines, 
if one knew a positive detection was made such that the assumed class contains the target that is present in the environment, one might ask which signal maximizes the probability of correctly deciding which target in the class is actually present. We consider these two issues related to designing waveforms that are optimal with respect to a target class further in Appendix B.

Next, in each formulation we designed signals that maximize the probability of detection based on the transmission and reception of a single pulse. However, a more practical situation is one where multiple pings are transmitted. Therefore, methods that accumulate the information garnered from each return to improve the quality of the decision, or even adapt the next transmission based on old information are naturally desirable[41, 42].

Finally, in all cases we assumed the noise and reverberation to be stationary and Gaussian. In real world marine settings noise and reverberation are likely to be nonstationary. Since time-frequency analysis has been shown to capture nonstationary effects a natural idea is to reformulate the detection problem in terms of some joint-time frequency representation $[43,44,45]$. Appendix $\mathrm{C}$ considers preliminary research directions related to this area in more detail. 


\section{APPENDIX A}

\section{RELATIONSHIP TO LINEAR PHASE AND MINIMUM PHASE SIGNALS AND SYSTEMS}

The linear phase, minimum duration, and maximum concentration signals discussed in Chapters 3 and 4 naturally evoke memories of linear phase and minimum phase systems, which are two common classes of linear systems, since we found that linear phase signals minimize duration while minimum phase systems are known to have impulse responses that possess the so-called minimum-delay property [27, pps. 248-250]. In this appendix we discuss some of the general properties of linear phase and minimum phase systems and relate them to the the linear phase, minimum duration, and maximum concentration signals derived in Chapters 3 and 4 . To do so we attribute the system property in question to the associated system impulse response and use it to compare to the signals derived in Chapters 3 and 4.

Since the minimum duration signal design was derived in a continuous setting, the connections between concepts familiar in discrete time systems are not immediately clear. However, one way to draw such connections is to discretize (3.8) and treat it as the impulse response of some discrete-time linear system. It can be shown [27, pps. 297-298] that causal Finite Impulse Response (FIR) systems have linear phase if they are symmetric or antisymmetric about the midpoint of the impulse response. With reference to Figure 9 (a), we see that not all linear phase signals derived according to the methods in 3.2.1 yield signals that are symmetric or anti-symmetric about the midpoint of the signal. It has also been shown [46] that causal Infinite Impulse Response (IIR) systems can have linear phase but, when they do, cannot be expressed as a rational transfer function, thereby making it impossible to 
implement in terms of a difference equation. Thus, we can conclude that the signal derived in 3.2.1 is not necessarily the impulse response associated with a rational transfer function, with the one exception being the case when (3.8) yields a discrete sequence that is symmetric or anti-symmetric about the sequence midpoint. If this is the case then it is also well known [27, pps. 306-307] that the zeros exist in conjugate reciprocal pairs, making it impossible for all of the zeros to be inside the unit circle.

Minimum phase is also a concept in DSP that has traits reminiscent of the notions discussed Chapters 3 and 4. In particular, minimum phase systems - those that have all of their poles and zeros inside the unit circle - possess a property known as minimum-delay. Mathematically, this property is described as

$$
\sum_{n=0}^{K}|h(n)|^{2} \leqslant \sum_{n=0}^{K}\left|h_{m p}(n)\right|^{2} \quad \forall K \in \mathbb{Z}
$$

where $h(n)$ is a member of the family of all impulse responses having the same magnitude spectrum, $|H(\omega)|^{2}$, and $h_{m p}(n)$ is the unique [27, pg. 280] member of this class exhibiting the minimum phase property. Intuitively, this property says that of all systems having the same magnitude spectrum, constraining the system to be minimum phase yields an impulse response that maximally concentrates its energy in lowest samples (cf. Figure 14). However, as shown by example in section 3.3, minimum phase signals do not have duration less than the the linear phase signal given by (3.8), an observation consistent with the fact that minimum phase signals cannot have linear phase. Finally, to draw a comparison between a minimum phase signal with specified magnitude spectrum and the signals designed in Chapter 4 we must let $\epsilon \rightarrow \infty$. But, as shown in section 4.3.4, this situation leads to the minimum duration solution, which, due to its linear phase, cannot be achieved by a minimum phase signal. 


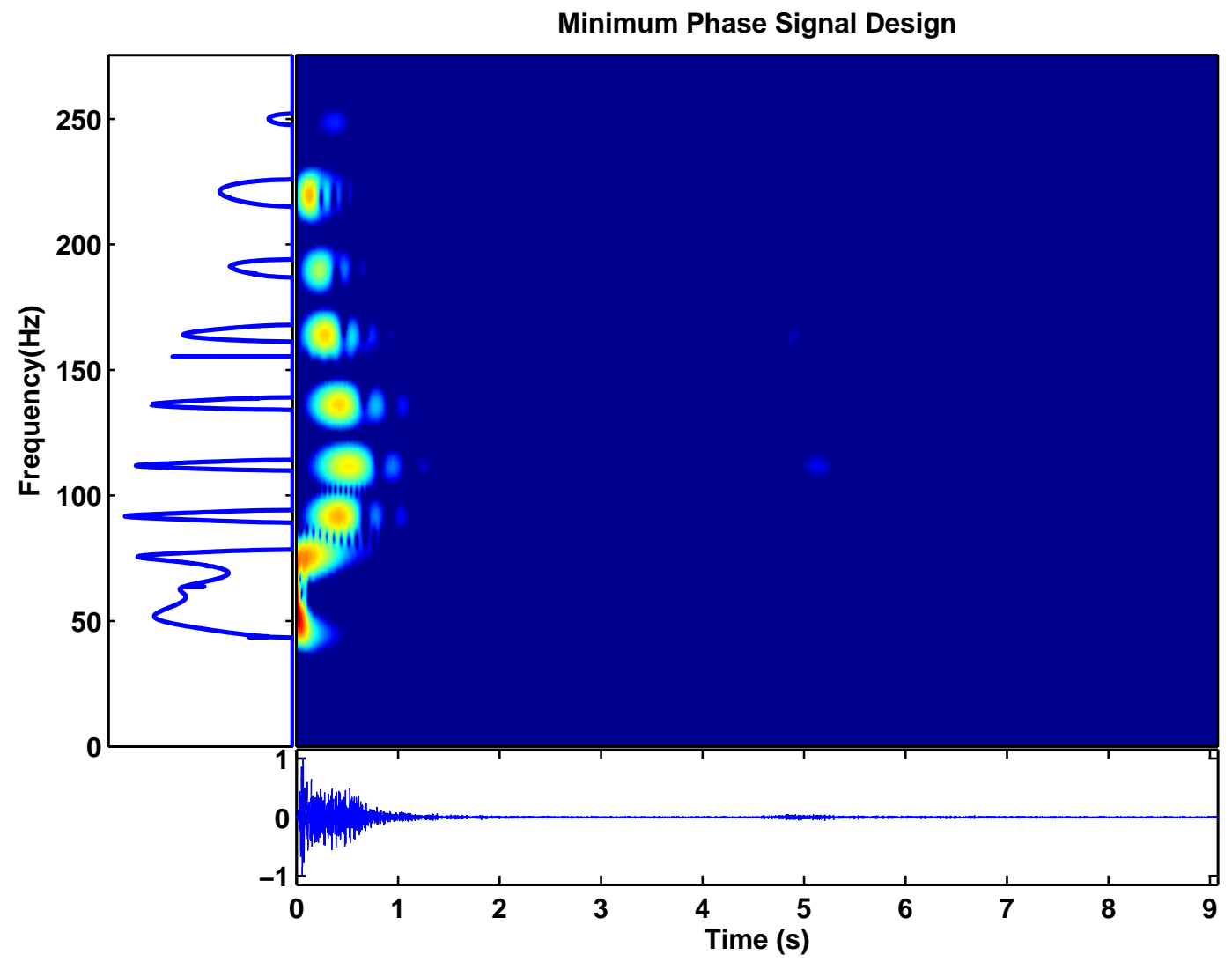

Figure 14: Spectrogram (Main Panel) Time Series (Lower Panel) and Magnitude Spectrum (Left Panel) of Minimum Phase solution exhibiting minimum delay property 


\section{APPENDIX B}

\section{MULTITARGET WAVEFORM DESIGN FOR OPTIMAL DETECTION AND CLASSIFICATION}

Throughout this thesis we assumed that there is only one potential target of interest present in the environment. In practice, it may be unrealistic to assume precise knowledge of a target we wish to detect and therefore more desirable to assume that the target present is a member of a class of potential targets. In this appendix we take this viewpoint and assume that if a target is present in the environment it is a member of a known set of candidates targets. From this point of view we develop two approaches that incorporate this target response uncertainty, but do so with differing assumptions. First, we design a transmit waveform that maximizes the minimum probability of detecting a target within a user-specified class of elastic targets based on the modeling assumptions given in Chapter 2 and summarized in Figure 1. Next, we assume that a positive detection has been made such that one of the targets within the assumed class is present and design a signal that maximizes the probability of correctly classifying the true target. In contrast to the first approach, we do not assume the presence of signal dependent noise. Rather, we assume all undesirable effects can be modeled as an Additive White Gaussian Noise (AWGN) noise process. 


\section{B.1 MULTITARGET WAVEFORM DESIGN FOR OPTIMAL DETECTION}

In Chapter 2 we found the waveform that maximizes the probability of detecting a single, fixed target for a given probability of false alarm, $P_{F A}$. The relationship between $P_{D}$ as a function of $P_{F A}$ and the magnitude spectrum of the transmit waveform, $B(f)$, is given by

$$
P_{D}=P_{F A}^{\frac{1}{1+d}}
$$

where

$$
d=\int_{-W / 2}^{W / 2} \frac{T B^{2}(f)|G(f)|^{2}}{P_{h}(f) T B^{2}(f)+P_{n}(f)} d f
$$

One way to generalize the target specific waveform design approach given by Kay to the class specific viewpoint proposed above is to design $B(f)$ that maximizes the minimum $d_{i}$ given as follows

$$
d_{i}=\int_{-W / 2}^{W / 2} \frac{T B^{2}(f)\left|G_{i}(f)\right|^{2}}{P_{h}(f) T B^{2}(f)+P_{n}(f)} d f
$$

where $\left|G_{i}(f)\right|^{2}$ is the magnitude spectrum of the $i^{t h}$ target response in the given target class. Since $P_{D}$ in ( B.1) is a monotonically increasing function of $d$ for each $P_{F A} \in(0,1)$, the $B(f)$ that maximizes the minimum $d_{i}$ will also maximize the minimum $P_{D}$ among all targets in the class for each $P_{F A}$. Mathematically, we can pose the problem in the form of the following constrained nonlinear program

$$
\begin{array}{r}
\max _{B(f)} \min _{i}\left\{d_{i}\left(B(f) ;\left|G_{i}(f)\right|^{2}\right)\right\} \\
\text { subject to } \int_{-W / 2}^{W / 2} B^{2}(f) d f \leq E \\
B(f) \geq 0
\end{array}
$$

where $i=1, \ldots, M$ and $M$ is the number of elements in the assumed class. 


\section{B.1.1 Initialization}

In order to solve the nonlinear program above we must first discretize all continuous components so that vector matrix notation can be used and the problem can be implemented on a digital computer. Following this step one must construct an initial solution that can be used to initialize an iterative algorithm, such as sequential quadratic programming (SQP), that solves ( B.4). One initialization approach that has been found to be efficient is to first determine the individual optimal solution for each potential target based on the theory presented in Chapter 2,

$$
\begin{aligned}
& y_{1}=T\left(B_{1}^{o p t}(f)\right)^{2}=\max \left(\frac{\lambda_{1}^{-1 / 2} \sqrt{P_{n}(f)\left|G_{1}(f)\right|^{2}}-P_{n}(f)}{P_{h}(f)}, 0\right) \\
& y_{2}=T\left(B_{2}^{o p t}(f)\right)^{2}=\max \left(\frac{\lambda_{2}^{-1 / 2} \sqrt{P_{n}(f)\left|G_{2}(f)\right|^{2}}-P_{n}(f)}{P_{h}(f)}, 0\right) \\
& \vdots \\
& y_{M}=T\left(B_{M}^{o p t}(f)\right)^{2}=\max \left(\frac{\lambda_{M}^{-1 / 2} \sqrt{P_{n}(f)\left|G_{M}(f)\right|^{2}}-P_{n}(f)}{P_{h}(f)}, 0\right)
\end{aligned}
$$

then find the scalar weights $a_{1}, \ldots, a_{M}$ that solve the following constrained nonlinear program

$$
\begin{array}{r}
\max _{a_{1}, \ldots, a_{m}} \min _{i}\left\{d_{i}\left(B_{0}(f) ;\left|G_{i}(f)\right|^{2}\right)\right\} \\
\text { subject to } \int_{-W / 2}^{W / 2} B_{0}^{2}(f) d f \leq E \\
B_{0}(f) \geq 0
\end{array}
$$

where $B_{0}(f)$ is a linear combination of individual solutions, defined as follows

$$
B_{0}(f)=a_{1} y_{1}+a_{2} y_{2}+\cdots+a_{M} y_{m}
$$

So, given a set of transmit waveforms, $y_{1}, \ldots, y_{M}$, that are each optimal for detecting a specific target in the class, solving ( B.6) amounts to finding the best linear combination of these waveforms that maximizes the minimum probability of detecting a target within the class. The utility of this problem formulation is that we reduce the dimensionality of the 
problem posed in ( B.4), which is equal to the potentially large number of frequency points used for discretization, to a problem whose dimension is equal to the total number of targets assumed to be in the class, $M$. Though suboptimal, the hope is that the solution is close enough to the optimal solution to lead to rapid convergence of an algorithm solving ( B.4).

\section{B.1.2 Simulation Results}

In this section we present some preliminary simulation results that test the approach outlined in the previous section. In Figures 15 and 16 we show the optimal solution that results from solving ( B.4) (black) and from solving ( B.6) (magenta) in terms of the optimal magnitude spectra and the associated detection performance for each of the $M=6$ targets in the assumed class. For comparison we also include the optimal transmit signal associated with each individual target in the class (red). In Figure 16 we see that the the approach appears to allocate energy in a way that seeks to maximize worst case performance.

We also point out that it appears that the M-dimensional initialization approach given by ( B.6) tends to perform almost just as well as the full solution obtained from ( B.4). Thus, we have a suboptimal solution ( B.6) that almost achieves the optimal solution ( B.4) for a fraction of the computational burden. 

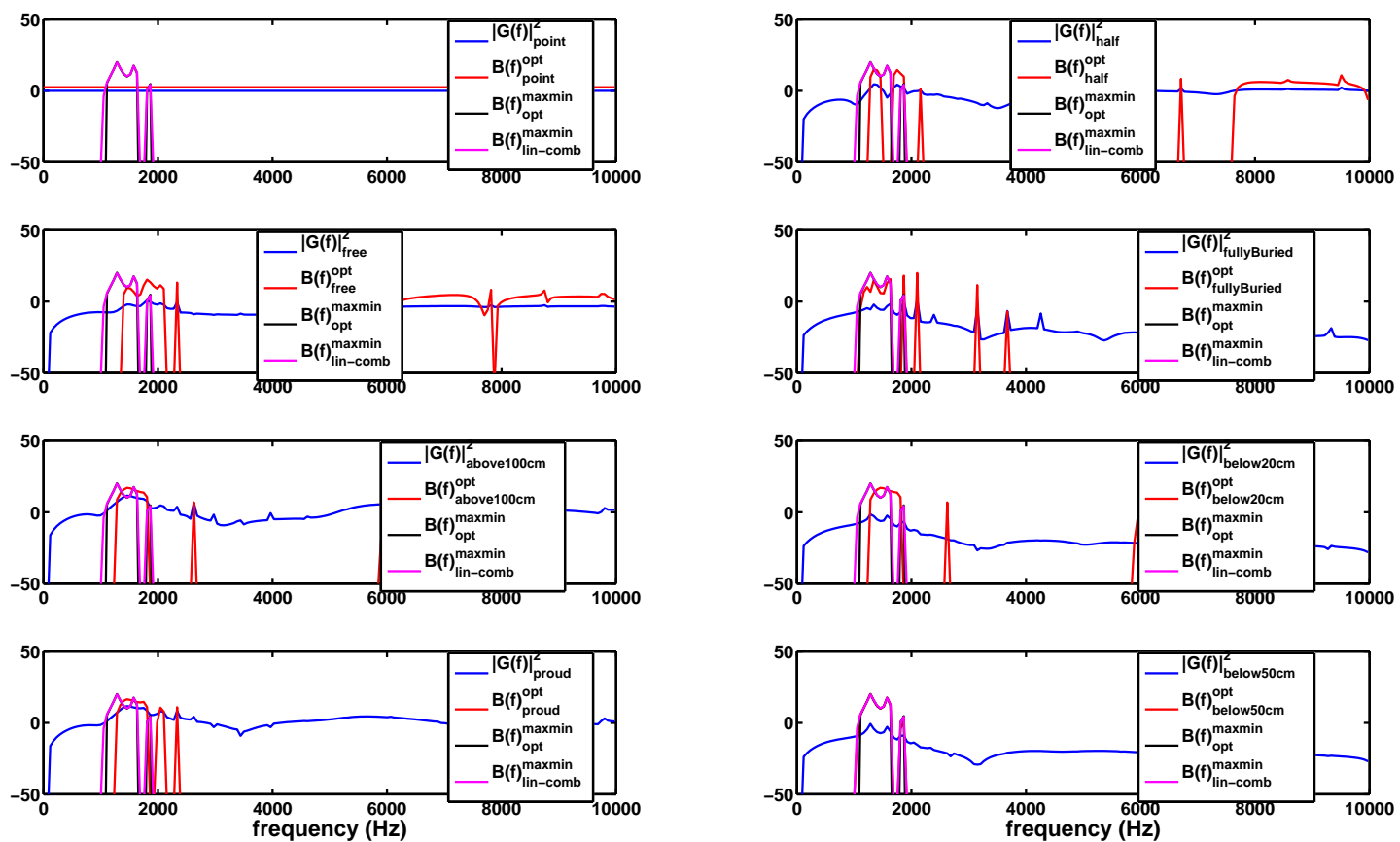

Figure 15: Spectra of Multitarget Waveform Design Approach 

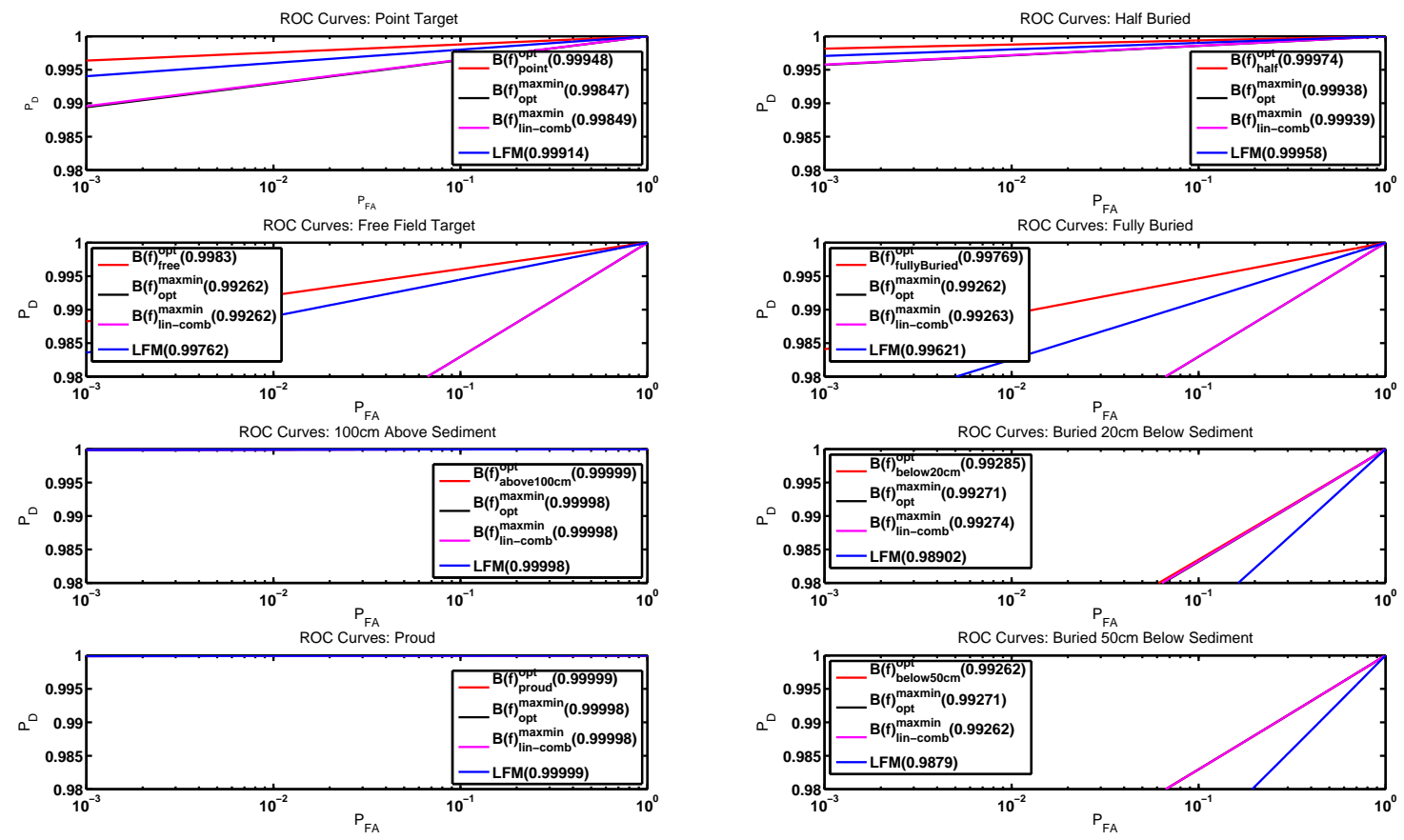

Figure 16: Performance of Multitarget Waveform Design Approach 


\section{B.2 MULTITARGET WAVEFORM DESIGN FOR OPTIMAL CLASSIFICATION}

In contrast to B.1, this section makes two different assumptions. First, we assume that all noise can be modeled as AWGN and therefore all interference is signal independent. Second, we assume that we know that a positive detection has been made which signals that we know that the environment contains a target that is a member of our assumed class. The goal is now to design a waveform that maximizes the probability of correctly deciding which of the targets from the class is actually present.

To design this waveform we adopt the Minimum Probability of Error (MPE) classifier (a/k/a Minimum Distance Receiver) [19, pps. 80-82, 119-121] structure and design signals that maximize the minimum deflection between classifier branch outputs. Each classifier branch output is given by

$$
D_{i}(x)=\left\|x-M_{i} s\right\|^{2}
$$

where $x$ is a vector of received data samples, $M_{i}$ is a convolution matrix constructed from the

impulse response of the $i^{\text {th }}$ target, and $s$ is a vector of time-domain samples of the transmitted waveform. Thus, the deflection coefficient associated with branches $j$ and $k$ assuming target $i$ is present is defined as

$$
d_{i k}^{i}=\frac{\left(E\left\{D_{i} \mid \text { Target } \mathrm{i}\right\}-E\left\{D_{j} \mid \text { Target i }\right\}\right)^{2}}{\operatorname{var}\left\{D_{i} \mid \text { Target i }\right\}+\operatorname{var}\left\{D_{j} \mid \text { Target i }\right\}}
$$

where $E\{\}$ and var \{\} denote the statistical expectation and variance operators, respectively, conditioned on the $i^{t} h$ target. After some lengthy calculations we arrive at the following

$$
\begin{aligned}
E\left\{D_{i} \mid \text { Target i }\right\} & =N \sigma_{w}^{2} \\
E\left\{D_{j} \mid \text { Target i }\right\} & =s^{T} Q_{i j} s+N \sigma_{w}^{2} \\
\operatorname{var}\left\{D_{i} \mid \text { Target i }\right\} & =2 N \sigma_{w}^{4} \\
\operatorname{var}\left\{D_{j} \mid \text { Target i }\right\} & =4 \sigma_{w}^{2} s^{T} Q_{i j} s+2 N \sigma_{w}^{4}
\end{aligned}
$$


Thus, ( B.9) simplifies to

$$
\begin{aligned}
d_{i j}^{i} & =\frac{\left(s^{T} Q_{i j} s\right)^{2}}{4 N \sigma_{w}^{4}+s^{T} Q_{i j} s} \\
& <s^{T} Q_{i j} s
\end{aligned}
$$

where $N$ is the total number of samples in the transmitted signal and received data vectors, $\sigma_{w}^{2}$ is the AWGN variance, and $Q_{i j}=\left(M_{i}-M_{j}\right)^{T}\left(M_{i}-M_{j}\right)$. Thus the signal design relevant to the classification problem in this section is given as the solution to the following nonlinear program

$$
\begin{gathered}
\max _{s} \min _{i} d_{i j}^{i}(s) \\
\text { subject to } s^{T} s \leq E
\end{gathered}
$$

As mentioned in Chapter 5, a number of other authors have considered similar approaches leading to ( B.16) or its variants. However, to the authors knowledge the literature is devoid of results ( B.10)-( B.13), which, combined with the definition of deflection given in ( B.9), gives a simple statistical justification for the objective function of ( B.16) within the context of the optimal MPE classifier architecture. 


\section{APPENDIX C}

\section{EXTENSIONS TO NONSTATIONARY ENVIRONMENTS}

In Chapter 2 we overviewed the main results of [4] wherein, under the assumption that all random processes are stationary and Gaussian, the optimal detector was derived. Based on this detector Kay determined how the detection performance was related to the transmit signal, which, strictly speaking, has nothing to do with the optimality of the detector. The mathematical link that allowed for the derivation of the optimal signal that maximizes probability of detection is

$$
\sigma_{0}^{2}=\int_{-W / 2}^{W / 2} \frac{T B^{2}(f)|G(f)|^{2}}{P_{h}(f) T B^{2}(f)+P_{n}(f)} d f
$$

since probability of detection is related to this quantity in the following way

$$
P_{D}=P_{F A}^{\frac{1}{1+\sigma_{0}^{2}}}
$$

So, choosing $|S(f)|^{2}$ to maximize ( C.1) will make ( C.2) as close as possible to 1 for a given probability of false alarm.

In a similar spirit as [4], but at this point without the same rigorous mathematical support, in future research we plan to consider the derivation of the Time-Frequency Distribution (TFD), $C_{s s}(t, f)$, that maximizes the following function

$$
\sigma_{0}^{2}=\int_{-T / 2}^{T / 2} \int_{-W / 2}^{W / 2} \frac{C_{s s}(t, f) C_{g g}(t, f)}{\bar{C}_{h h}(t, f) C_{s s}(t, f)+\bar{C}_{n n}(t, f)} d t d f
$$


subject to the constraint that $\int_{-T / 2}^{T / 2} \int_{-W / 2}^{W / 2} C_{s s}(t, f)=E$ where $C_{s s}(t, f)$ and $C_{g g}(t, f)$ are TFDs associated with the transmitted waveform and the target response, respectively, while $\bar{C}_{h h}(t, f)$ and $\bar{C}_{n n}(t, f)$ are the Time-Frequency Spectra ${ }^{1}$ of the channel and additive noise random processes, respectively. Based on very cursory and preliminary analyses it seems that an expression close to the following

$$
C_{\text {opt }}(t, f)=\max \left(\frac{\lambda^{-1 / 2} \sqrt{\bar{C}_{n n}(t, f)}-\bar{C}_{n n}(t, f)}{\bar{C}_{h h}(t, f)}, 0\right)
$$

will maximize ( C.3) if we assume that each TF Spectrum is non-negative. It is thought that such a solution will yield an transmit signal design that optima for detection in a non-stationary environment. Clearly, this expression bears a striking resemblance to ( C.1) and the derivation that leads to it is what motivates our belief that ( C.4) will maximize ( C.3). Related work in the are of optimal detection in nonstationary environments appears in $[47,44,43,48,45]$

\footnotetext{
${ }^{1}$ defined as the expected value of the TFD of the associated random process
} 


\section{BIBLIOGRAPHY}

[1] P. M. Woodward, Probability and Information Theory, with Applications to Radar. McGraw-Hill Book Co., Inc., 1953.

[2] M. Bell, "Information theory and radar waveform design," IEEE Trans. Inf. Theory, vol. 39, pp. 1578-1597, September 1993.

[3] Y. Yang and R. Blum, "MIMO radar waveform design based on mutual information and minimum mean-squared error estimation," IEEE Trans. Aerospace and Electronic Systems, vol. 43, no. 1, pp. 330-343, 2007.

[4] S. Kay, "Optimal signal design for detection of gaussian point targets in stationary gaussian clutter/reverberation," IEEE J. Sel. Topics in Sig. Proces. Mag., vol. 1, pp. 3141, June 2007.

[5] S. Kay, "Waveform design for multistatic radar detection," IEEE Trans. Aerospace and Electronic Systems, vol. 45, pp. 1153-1166, July 2009.

[6] J. Li, J. R. Guerci, and L. Xu, "Signal waveform's optimal-under-restriction design for active sensing," IEEE Signal Processing Letters, vol. 13, September 2006.

[7] A. Papandreou-Suppappola, A. Nehorai, and R. Calderbank, "Waveform agility in radar systems [special issue]," IEEE Signal Processing Magazine, vol. 26, January 2009.

[8] J. J. Zhang, A. Papandreou-Suppappola, B. Gottin, and C. Ioana, "Time-frequency characterization and receiver waveform design for shallow water environments," IEEE Trans. Sig. Proc., vol. 57, August 2009.

[9] S. U. Pillai, H. S. Oh, D. C. Youla, and J. R. Guerci, "Optimal transmit-receiver design in the presence of signal-dependent interference and channel noise," IEEE Trans. Inf. Theory, vol. 46, pp. 577-584, March 2000.

[10] L. Jackson, S. Kay, and N. Vankayalapati, "Iterative method for nonlinear fm synthesis of radar signals," Aerospace and Electronic Systems, IEEE Transactions on, vol. 46, no. 2, pp. 910-917, 2010.

[11] L. Patton and B. Rigling, "Modulus constraints in adaptive radar waveform design," in Radar Conference, 2008. RADAR'08. IEEE, pp. 1-6, IEEE, 2008. 
[12] L. Patton, On the satisfaction of modulus and ambiguity function constraints in radar waveform optimization for detection. PhD thesis, Wright State University, 2009.

[13] B. Hamschin and P. Loughlin, "Optimal time and frequency domain waveform design for target detection," in Proceedings of SPIE, vol. 7696, p. 76960F, 2010.

[14] D. Slepian and H. O. Pollak, "Prolate spheroidal wave functions, fourier analysis and uncertainty, i,," Bell Syst. Tech. J., vol. 40, pp. 43-64, 1961.

[15] H. J. Landau and H. O. Pollak, "Prolate spheroidal wave functions, fourier analysis and uncertainty, ii," Bell Syst. Tech. J., vol. 40, pp. 65-84, 1961.

[16] H. J. Landau and H. O. Pollak, "Prolate spheroidal wave functions, fourier analysis and uncertainty, iii," Bell Syst. Tech. J., vol. 41, pp. 1296-1336, 1962.

[17] D. Slepian, "Prolate spheroidal wave functions, fourier analysis and uncertainty, iv," Bell Syst. Tech. J., vol. 43, pp. 3009-3058, 1964.

[18] W. Au, A. Popper, and R. Fay, Hearing by whales and dolphins. Springer Verlag, 2000.

[19] S. Kay, Fundamentals of Statstical Signal Processing: Detection Theory, vol. 2 of Prentice Hall Signal Processing Series. Prentice Hall, 1998.

[20] H. Van Trees, "Optimum signal design and processing for reverberation-limited environments," Military Electronics, IEEE Transactions on, vol. 9, no. 3, pp. 212-229, 1965.

[21] R. Romero and N. Goodman, "Waveform design in signal-dependent interference and application to target recognition with multiple transmissions," Radar, Sonar \& Navigation, IET, vol. 3, no. 4, pp. 328-340, 2009.

[22] L. Flax, L. R. Dragonette, and H. überall, "Theory of elastic resonance excitation by sound scattering," The Journal of the Acoustical Society of America, vol. 63, no. 3, pp. 723-731, 1978.

[23] G. C. Gaunaurd and D. Brill, "Acoustic spectrogram and complex-frequency poles of a resonantly excited elastic tube," The Journal of the Acoustical Society of America, vol. 75, no. 6, pp. 1680-1693, 1984.

[24] G. Gaunaurd, "Transient acoustic scattering by fluid-loaded elastic shells," International Journal of Solids and Structures, vol. 27, no. 6, pp. 699-711, 1991.

[25] G. C. Gaunaurd and H. C. Strifors, "Frequency- and time-domain analysis of the transient resonance scattering resulting from the interaction of a sound pulse with submerged elastic shells," Ultrasonics, Ferroelectrics and Frequency Control, IEEE Transactions on, vol. 40, no. 4, pp. 313-324, 1993. 
[26] C. Y. Tsui, G. N. Reid, and G. C. Gaunaurd, "Resonance scattering by elastic cylinders and their experimental verification," The Journal of the Acoustical Society of America, vol. 80, no. 2, pp. 382-390, 1986.

[27] A. Oppenheim, R. Schafer, and J. Buck, Discrete-Time Signal Processing. Prentice Hall, 1999.

[28] L. Cohen, Time-Frequency Analysis. Prentice Hall Signal Processing Series, Prentice Hall, 1995.

[29] H. Stark and Y. Yang, Vector Space Projections - A Numerical Approach to Signal and Image Processing, Neural Nets, and Optics. John Wiley \& Sons, Inc., 1998.

[30] J. O. Smith, Introduction to Digital Filters with Audio Applications. http://ccrma.stanford.edu/ jos/filters/, accessed (April 4, 2011). online book.

[31] D. B. Percival and A. T. Walden, Spectral analysis for physical applications. Cambridge University Press, 1993.

[32] D. Slepian, "Some comments on fourier analysis, uncertainty, and modeling," SIAM Review, vol. 25, pp. 379-393, 1983.

[33] P. Loughlin and L. Cohen, "The uncertainty principle: global, local, or both?," IEEE Trans. Sig. Proc., vol. 52, no. 5, pp. 1218-1277, 2004.

[34] D. J. Thomson, "Spectrum estimation and harmonic analysis," Proceedings of the IEEE, vol. 70, pp. 1055-1096, 1982.

[35] R. Courant and D. Hilbert, Methods of mathematical physics. Wiley-Interscience, 1989.

[36] P. Stoica and R. Moses, Spectral analysis of signals. Pearson/Prentice Hall, 2005.

[37] The Math Works, Inc., 24 Prime Park Way, Natick, MA 01760-1500, Optimization Toolbox User's Guide Version 5, 5.1 ed., September 2002.

[38] J. Nocedal and S. Wright, Numerical optimization. Springer verlag, 1999.

[39] J. Guerci and S. Pillai, "Adaptive transmission radar: the next wave?," in National Aerospace and Electronics Conference, 2000. NAECON 2000. Proceedings of the IEEE 2000, pp. 779-786, IEEE, 2000.

[40] D. Garren, M. Osborn, A. Odom, J. Goldstein, S. Pillai, and J. Guerci, "Optimal transmission pulse shape for detection and identification with uncertain target aspect," in Radar Conference, 2001. Proceedings of the 2001 IEEE, pp. 123-128, IEEE, 2001. 
[41] N. Goodman, P. Venkata, and M. Neifeld, "Adaptive waveform design and sequential hypothesis testing for target recognition using cognitive radar," IEEE J. Sel. Top. Signal Process, vol. 1, pp. 105-113, 2007.

[42] A. Wald, Sequential Analysis. Dover, 2004.

[43] A. Sayeed and D. Jones, "Optimal detection using bilinear time-frequency and time-scale representations," Signal Processing, IEEE Transactions on, vol. 43, no. 12, pp. 28722883, 1995.

[44] P. Flandrin, "A time-frequency formulation of optimum detection," Acoustics, Speech and Signal Processing, IEEE Transactions on, vol. 36, no. 9, pp. 1377-1384, 1998.

[45] G. Matz and F. Hlawatsch, "Time-frequency formulation and design of optimal detectors," in Time-Frequency and Time-Scale Analysis, 1996., Proceedings of the IEEE-SP International Symposium on, pp. 213-216, IEEE, 1996.

[46] M. Clements and J. Pease, "On causal linear phase IIR digital filters," Acoustics, Speech and Signal Processing, IEEE Transactions on, vol. 37, no. 4, pp. 479-484, 1989.

[47] W. Martin and P. Flandrin, "Wigner-ville spectral analysis of nonstationary processes," Acoustics, Speech and Signal Processing, IEEE Transactions on, vol. 33, no. 6, pp. 1461$1470,1985$.

[48] P. Loughlin and B. M. Hamschin, "Detection with the wigner distribution of a pulse propagating with dispersion and damping," Journal of Modern Optics, p. (submitted), 2011. 